OPEN ACCESS

Edited by:

Hansen Wang,

University of Toronto, Canada

Reviewed by:

Richardson N. Leão,

Karolinska Institute (KI), Sweden

Diasynou Fioravante,

University of California, Davis,

United States

*Correspondence:

J. Gerard G. Borst

g.borst@erasmusmc.nl

tPresent address:

Tiantian Wang,

Janssen Pharmaceutica NV.

Beerse, Belgium

Received: 22 July 2017 Accepted: 12 December 2017

Published: 04 January 2018

Citation:

Wang T, van Woerden GM, Elgersma Y and Borst JGG (2018) Enhanced Transmission at the Calyx of Held Synapse in a Mouse Model for Angelman Syndrome.

Front. Cell. Neurosci. 11:418. doi: 10.3389/fncel.2017.00418

\section{Enhanced Transmission at the Calyx of Held Synapse in a Mouse Model for Angelman Syndrome}

\author{
Tiantian Wang ${ }^{1 \dagger}$, Geeske M. van Woerden ${ }^{1,2}$, Ype Elgersma ${ }^{1,2}$ and J. Gerard G. Borst ${ }^{1 *}$ \\ 1 Department of Neuroscience, Erasmus MC, University Medical Center Rotterdam, Rotterdam, Netherlands, ${ }^{2}$ ENCORE \\ Expertise Center for Neurodevelopmental Disorders, Erasmus MC, University Medical Center Rotterdam, Rotterdam, \\ Netherlands
}

The neurodevelopmental disorder Angelman syndrome (AS) is characterized by intellectual disability, motor dysfunction, distinct behavioral aspects, and epilepsy. AS is caused by a loss of the maternally expressed UBE3A gene, and many of the symptoms are recapitulated in a Ube3a mouse model of this syndrome. At the cellular level, changes in the axon initial segment (AIS) have been reported, and changes in vesicle cycling have indicated the presence of presynaptic deficits. Here we studied the role of UBE3A in the auditory system by recording synaptic transmission at the calyx of Held synapse in the medial nucleus of the trapezoid body (MNTB) through in vivo whole cell and juxtacellular recordings. We show that MNTB principal neurons in Ube3a mice exhibit a hyperpolarized resting membrane potential, an increased action potential (AP) amplitude and a decreased AP half width. Moreover, both the pre- and postsynaptic AP in the calyx of Held synapse of Ube3a mice showed significantly faster recovery from spike depression. An increase in AIS length was observed in the principal MNTB neurons of Ube3a mice, providing a possible substrate for these gain-of-function changes. Apart from the effect on APs, we also observed that EPSPs showed decreased shortterm synaptic depression (STD) during long sound stimulations in AS mice, and faster recovery from STD following these tones, which is suggestive of a presynaptic gain-offunction. Our findings thus provide in vivo evidence that UBE3A plays a critical role in controlling synaptic transmission and excitability at excitatory synapses.

Keywords: Ube3a, synaptic transmission, synaptic morphology, juxtacellular recording, short-term plasticity, axon initial segment, mouse model, action potential

\section{INTRODUCTION}

Angelman syndrome (AS) is a severe neurodevelopmental disorder caused by loss of the maternal allele of the UBE3A gene. AS has an incidence of 1:25,000 and affects both genders equally (Mertz et al., 2013). The disorder is associated with intellectual disability, profound speech impairment, motor abnormalities, epilepsy, and specific behavioral abnormalities including autism and impaired sleep rhythm (reviewed in Buiting et al., 2016). UBE3A encodes the ubiquitin ligase E3A (UBE3A; also, termed E6-associated protein, E6-AP), which is expressed solely from the maternal allele in mature neurons. Ubiquitin ligase E3A covalently attaches polyubiquitin chains to proteins, resulting in either their degradation by the $26 \mathrm{~S}$ proteasome or an impact on their 
activity. Although several substrates have been identified, it is not clear to what extent these targets contribute to the AS phenotype.

Heterozygous mice with a maternally inherited Ube3a mutation show phenotypes that are similar to AS patients, including motor, cognitive and behavioral abnormalities (Jiang et al., 1998; Huang et al., 2013; Bruinsma et al., 2015; Silva-Santos et al., 2015). The AS mouse model also shows increased propensity for audiogenic seizures (Jiang et al., 1998; Van Woerden et al., 2007; Silva-Santos et al., 2015). At the cellular level pyramidal neurons in hippocampal area CA1 showed a more negative membrane potential and larger action potentials (AP) plus a lower AP threshold (Kaphzan et al., 2011). This was accompanied by an increased length of the axon initial segment (AIS) and increased expression of Nav1.6 voltage-dependent sodium channels, the $\alpha 1$ subunit of $\mathrm{Na}^{+} / \mathrm{K}^{+}$ATPase ( $\left.\alpha 1-\mathrm{NaKA}\right)$, and ankyrin-G, an anchoring protein of the AIS. It was hypothesized that the increased sodium channel expression was a homeostatic adaptation to counteract the decrease in excitability due to the more negative resting membrane potential; reduction of $\alpha 1-\mathrm{NaKA}$ expression indeed resulted in normalization of the sodium channel expression (Kaphzan et al., 2013). In addition to these postsynaptic effects, UBE3A also affects presynaptic signaling. It is enriched in axon terminals (Burette et al., 2017) and UBE3A may be involved in the vesicle cycle, since Ube3a mice show a reduction in the strength of inhibitory transmission onto neocortical pyramidal neurons, which was accompanied by a decrease in the number of synaptic vesicles, and a large increase in the number of clathrincoated vesicles (Wallace et al., 2012). More recently, it was shown that $u b e 3 a$ mutants showed endocytosis defects in the Drosophila neuromuscular junction leading to an increased number of boutons and increased synaptic depression ( $\mathrm{Li}$ et al., 2016).

To what extent these changes in functional neuronal properties are present in vivo is unknown. The principal aim of this work was to understand the physiological consequences of the Ube3a mutation for excitatory synaptic transmission, by studying the role of UBE3A in the auditory pathway. By recording synaptic transmission at the calyx of Held synapse in the medial nucleus of the trapezoid body (MNTB), critical parameters of pre- and postsynaptic function can be assessed in vivo. The calyx of Held synapse has a relay function in the auditory brainstem; each principal MNTB neuron is contacted by a single, giant terminal from a globular bushy cell of the contralateral AVCN. The principal neurons provide inhibition that is both well timed and sustained to many other auditory nuclei (Borst and Soria van Hoeve, 2012). Because of its large size, both pre- and postsynaptic activity can be recorded in vivo with a single pipette (Guinan and Li, 1990). Through waveform analysis of the characteristic extracellular waveform that can be recorded during juxtacellular recordings, estimates for synaptic delay, synaptic strength and postsynaptic excitability can be conveniently obtained (Lorteije and Borst, 2011). We found that $U b e 3 a$ mice show significantly fewer postsynaptic failures, as well as faster recovery from AP depression and faster recovery from prespike depression. In addition, the Ube3a mice also showed reduced short-term synaptic depression during tone stimuli and faster recovery from synaptic depression, providing in vivo evidence for changes in excitatory synaptic transmission.

\section{MATERIALS AND METHODS}

\section{Animals and Experiments}

Experiments were conducted in accordance with the European Communities Council Directive and were approved by the Animal Ethics Committee of the Erasmus MC.

Generation of the Ube3am-/p+ knock-out mutant (RRID: MGI:2181811; referred to as Ube3a $a^{\text {Exon3 }}$ mice), which carries a deletion of exon 3 of isoform 3 of Ube3a (ENSMUST00000107537, NM_001033962), has been described previously (Jiang et al., 1998). This line was maintained (>40 generations) in the 129S2/SvPasCrl background (Charles River) by breeding heterozygous $U b e 3 a^{m+/ p-}$ males with wild-type females. For the electrophysiology experiments, we crossed Ube $3 a^{m+/ p-}$ female Ube $3 a$ mutants with wild-type C57BL/6J (Charles River) males, to generate heterozygous Ube $3 a$ $\left(U b e 3 a^{m-/ p+}\right)$ mutants and littermate controls in the F1 hybrid 129S2-C57BL/6 background.

For the experiments with mice in the C57BL/6J background we used Ube3a mutants carrying an E113X nonsense mutation in exon 5 (ENSMUST00000200758.3, NM_011668.2) of the maternally inherited full length (isoform 2) Ube3a gene. This Ube $3 a^{E 113 X}$ mouse mutant (RRID: MGI: 5911277) was generated as follows (Figure 1A): the Ube3a genomic sequence (ENSMUSG00000025326) was obtained from Ensembl and used to design the primers for the targeting constructs. PCR fragments encompassing exon 5 using $5^{\prime}$ primer: $5^{\prime}$-CCGCGGG CTCCACTAGTCAATTTC-3' and 3'primer: 5'-GCGGCCGC ACCACAGTCCCTGGAGTTC-3' (4.9 kb; exon denotation according to ENSMUSG00000025326) and exon 6 using $5^{\prime}$ primer: 5'-GGCCGGCCGGAACTACCATATCCTGTTTTAC-3' and $3^{\prime}$ primer: 5'-GCGGCCGCAGCCGATCTAGGTATTC' $(4.6 \mathrm{~kb})$ were amplified using High Fidelity Taq Polymerase (Roche) on ES cell genomic DNA. Exon 5 and 6 were sequenced to verify that no mutations were introduced. To obtain a premature stop mutation at the end of exon 5 (p.Glu113Stop followed by a p.Gly114Leu mutation) the QuickChange site-directed mutagenesis kit (Stratagene) was used using $5^{\prime}$ primer: $5^{\prime}$-GATAAAAATGAACAAGAAGTGACT AAAAGATTTTAAAGGTAAGAG-3' and $3^{\prime}$ primer: $5^{\prime}$-CTC TTACCTTTAAAATCTTTTAGTCACTTCTTGTTCATTTTTA TC- $3^{\prime}$, and the PCR product was sequence verified. Both the $5^{\prime}$ flank with the mutation in exon 5 and the $3^{\prime}$ flank containing exon 6 were cloned on either side of a Neomycin-stop cassette flanked by loxP sites. For counterselection, the diphtheria toxin chain A (DTA) gene was inserted at the $5^{\prime}$ of the targeting construct. The targeting construct was linearized and electroporated into E14 ES cells (derived from 129P2 mice). Cells were cultured in BRL cell-conditioned medium in the presence of leukemia inhibitory factor. After selection with G418 $(200 \mu \mathrm{g} / \mathrm{ml})$, targeted clones were identified by PCR (long-range PCR from neomycin 


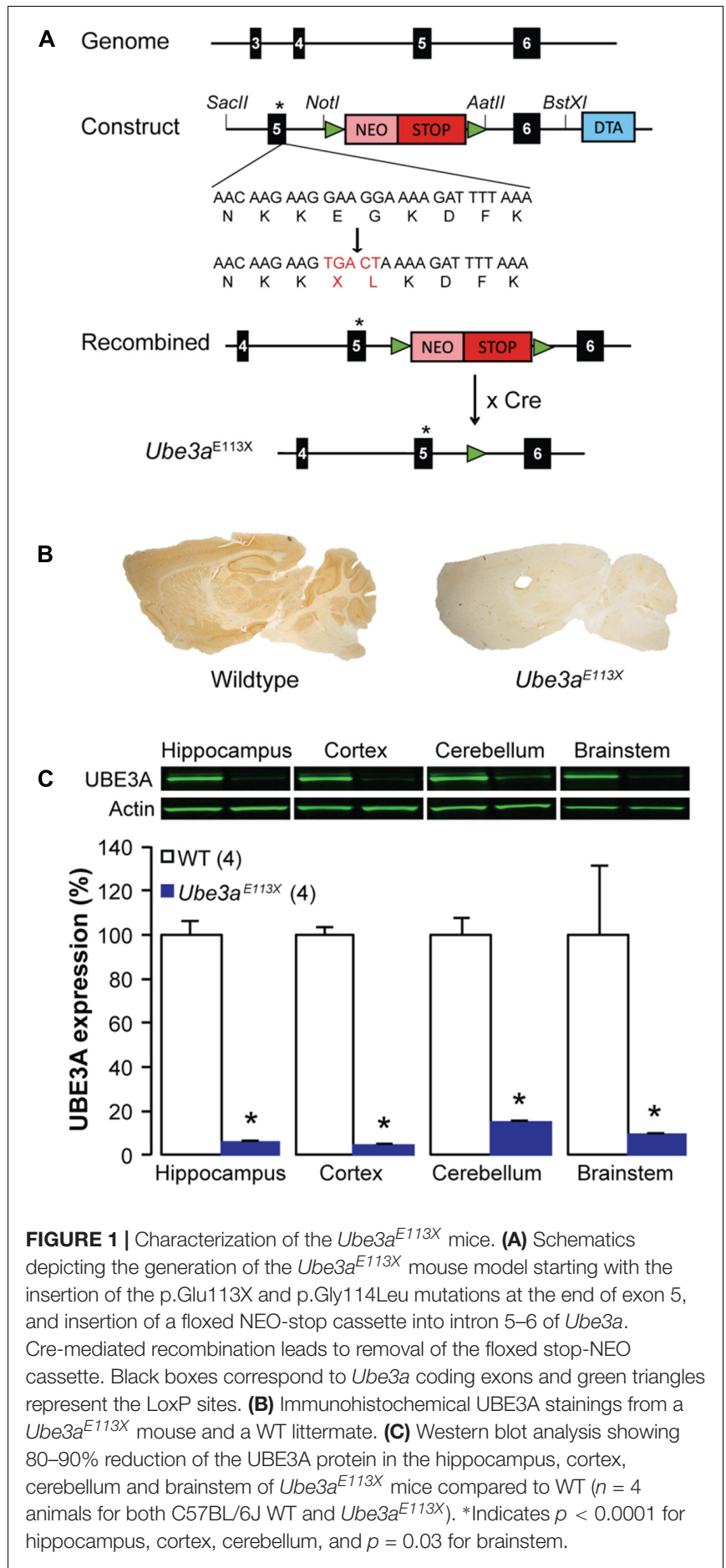

resistance gene to the region flanking the targeted sequence). A clone with verified karyotype was injected into blastocysts of C57BL/6 mice. Male chimeras were crossed with female $\mathrm{C} 57 \mathrm{BL} / 6 \mathrm{~J}$ mice. The resulting heterozygous offspring were crossed with TgCAG-cre mice (RRID: MGI:2176435) to delete the neomycin gene and stop cassette (Figure 1A). Ube $3 a^{E 113 X}$ offspring in which the neomycin cassette was removed and the Cre transgene was absent was used for subsequent crossings with
C57BL/6J mice. The Ube $3 a^{E 113 X}$ mice used for this study were crossed minimally 12 times into C57BL/6J.

The in vivo juxtacellular recordings were performed in mice of either hybrid 129S2-C57BL/6J background or C57BL/6J background. For the analysis of recovery from presynaptic AP depression and recovery from postsynaptic AP depression, data from both genetic backgrounds were obtained. For the analysis of recovery from EPSP depression, only the data from C57BL/6J background was included, since neurons of hybrid 129S2-C57BL/6J background exhibited very little shortterm depression (STD), probably owing to the observed high spontaneous firing rates (Table 1). Auditory Brainstem Response (ABR) recordings, in vivo whole-cell recordings, slice recordings, immunohistochemistry and quantification of AIS length were performed in Ube $3 a^{E 113 X}$ mice or litter mate controls in the C57BL/6J background.

\section{Auditory Brainstem Responses}

Auditory Brainstem Response recordings were performed as described previously (Nagtegaal et al., 2012). Briefly, the mice were anesthetized with a mixture of ketamine/xylazine $(60 / 10 \mathrm{mg} / \mathrm{kg})$ i.p. and placed in a sound-attenuated box in front of a loudspeaker (Radio Shack Super Tweeter 40-1310B), which presented tone pip stimuli ( $1 \mathrm{~ms}$ duration, $0.5 \mathrm{~ms}$ cosine-squared ramps, alternating polarity, repetition rate 80 per second) at a sound pressure level (SPL; re $20 \mu \mathrm{Pa}$ ) between -10 and $110 \mathrm{~dB}$. Needle electrodes were positioned subdermally at the base of both pinnae; the reference electrode was placed at the vertex, and a ground electrode near the sacrum. For determining ABR thresholds, 500 responses with artifacts $<30 \mu \mathrm{V}$ were averaged. Hearing level thresholds were measured at 4, 8, 16, and $32 \mathrm{kHz}$. Thresholds were defined as the lowest SPL ( $5 \mathrm{~dB}$ resolution) at which a reproducible peak (usually peak II or IV) was still present in either ear.

\section{Surgery and in Vivo Recordings}

After brief exposure to isoflurane, the adult mice (30 to 60 days old) were injected intraperitoneally with a ketamine-xylazine mixture (65 and $10 \mathrm{mg} / \mathrm{kg}$, respectively). A homothermic blanket (FHC, Bowdoinham, ME, United States) was used to keep the rectal body temperature at $36-37^{\circ} \mathrm{C}$. Animals were supine positioned, and a tracheotomy was performed so that the animal could be ventilated mechanically with oxygen (MiniVent; type 845; Harvard Apparatus). The animal was ventilated at a frequency of 150/min; with a stroke volume of $7 \mu \mathrm{l} / \mathrm{g}$ body weight. The right anterior inferior cerebellar artery and basilar artery were used as landmarks to locate the right MNTB, as previously described (Rodríguez-Contreras et al., 2008; Crins et al., 2011). Prior to recording, the dura and pia were removed to expose the brain surface. Ringer solution containing (in $\mathrm{mM}$ ): $\mathrm{NaCl} 135, \mathrm{KCl} 5.4, \mathrm{MgCl}_{2} 1, \mathrm{CaCl}_{2}$ 1.8, Hepes 5 (pH 7.2 with $\mathrm{NaOH}$ ) was applied to keep the brain surface moist.

In vivo juxtacellular and whole cell recordings were made from the principal neurons using thick-walled borosilicate glass micropipettes with filament, as described previously (Lorteije 
TABLE 1 | Comparison of spontaneous firing and complex waveforms between WT and Ube3a mice.

\begin{tabular}{|c|c|c|c|c|c|c|}
\hline Parameters (juxtacellular) & C57BL/6JWT & Ube3a $a^{E 113 X}$ & $p$-value & Hybrid WT & Ube3a Exon3 & $p$-value \\
\hline Spontaneous failures (\%) & $10.6 \pm 6.4$ & $1.4 \pm 1.0$ & 0.026 & $21.7 \pm 5.8$ & $4.0 \pm 2.9$ & 0.01 \\
\hline Spontaneous frequency $(\mathrm{Hz})$ & $26 \pm 6$ & $37 \pm 9$ & 0.34 & $58 \pm 4$ & $57 \pm 5$ & 0.82 \\
\hline Prespike-eEPSP delay (ms) & $0.33 \pm 0.01$ & $0.31 \pm 0.01$ & 0.41 & $0.3 \pm 0.02$ & $0.34 \pm 0.02$ & 0.17 \\
\hline eEPSP-eAP delay (ms) & $0.28 \pm 0.02$ & $0.23 \pm 0.01$ & 0.03 & $0.34 \pm 0.02$ & $0.27 \pm 0.01$ & 0.003 \\
\hline eAP halfwidth (ms) & $0.24 \pm 0.01$ & $0.27 \pm 0.01$ & 0.11 & $0.31 \pm 0.01$ & $0.29 \pm 0.01$ & 0.21 \\
\hline
\end{tabular}

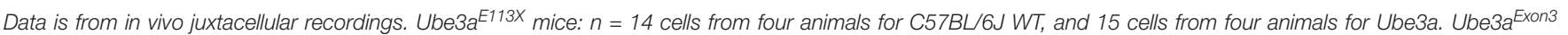

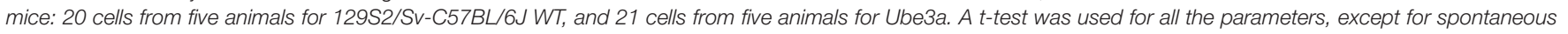
failures, for which Mann-Whitney $U$ test was used.

et al., 2009). The recording pipette was filled with Ringer solution for juxtacellular recording, and with intracellular solution (K-Gluconate 126, KCL 20, Na2-phosphocreatine 10, Mg-ATP 4, $\mathrm{Na}_{2}$-GTP 0.3, EGTA 0.5, HEPES 10; pH 7.2 with $\mathrm{KOH}$ ) for whole cell recording. Potentials were corrected for a junction potential of $-11 \mathrm{mV}$. The recording pipette penetrated the brain surface with a positive pressure of 300 mBar. The pressure was reduced to $\sim 30 \mathrm{mBar}$ after passing the brain surface, and to $0 \mathrm{mBar}$ when recording started.

To measure the membrane resistance during in vivo wholecell experiments we applied a $-10 \mathrm{mV}$ voltage step in wholecell voltage clamp configuration from a holding potential of $-70 \mathrm{mV}$. Spontaneous events were blanked before averaging (Figure 5A). To estimate the membrane resistance, the series resistance, which was calculated from the size of the negative peak at the beginning of the step, was subtracted from the input resistance, which was calculated from the steady-state hyperpolarizing current.

Data were acquired with a MultiClamp 700B patchclamp amplifier and pCLAMP 9.2 software (MDS Analytical Technologies, RRID:SCR_011323) at $50 \mathrm{kHz}$ with a 16-bit A/D converter (Digidata 1322A) after filtering at $10 \mathrm{kHz}$ (eight-pole Bessel filter).

\section{Auditory Stimulation}

Closed field sound stimulation was presented as described previously (Tan and Borst, 2007). A speaker probe was inserted into the left ear canal and was stabilized with silicone elastomer. A 2-noise-burst stimulation protocol was designed in MATLAB (Version R2008a), and the auditory stimulus was generated by Tucker Davis Technologies hardware (TDT, system 3, RX6 processor, PA5.1 attenuator, ED1 electrostatic driver, EC1 electrostatic speaker). A MATLAB program controlled both Clampex acquisition and auditory stimulation. The auditory stimulation protocol consisted of a $200 \mathrm{~ms}$ silent period, followed by two $400 \mathrm{~ms}$ bursts of wide band noise (bandwidth 2-40 kHz; $80 \mathrm{~dB} \mathrm{SPL}$ ), which were presented at six different intervals $(40,80,160,320,640,1280 \mathrm{~ms})$, followed by a silent period for a total sweep duration of $4 \mathrm{~s}$. Sound intensities were calibrated as previously described (Tan and Borst, 2007). Experiments were performed in a single-walled sound attenuated chamber (Gretch-Ken Industries).

\section{Preparation of Slices}

Adult mice (4-8 weeks old) were anesthetized with isoflurane and decapitated, after which the brain was taken out quickly and submerged in ice-cold carbogenated $\left(95 \% \mathrm{O}_{2}, 5 \% \mathrm{CO}_{2}\right)$ solution containing (in $\mathrm{mM}$ ): $93 \mathrm{NMDG}, 2.5 \mathrm{KCl}, 0.5 \mathrm{CaCl}_{2}$, 25 glucose, $10 \mathrm{MgCl}_{2}$, 20 HEPES, $1.25 \mathrm{NaH}_{2} \mathrm{PO}_{4}, 30 \mathrm{NaHCO}_{3}$, 2 thiourea, 5 sodium ascorbate, 3 Na-pyruvate, $\mathrm{pH} 7.4$ with $\mathrm{HCl}$ (Ting et al., 2014). Coronal slices containing the MNTB were cut in this solution at a thickness of $120 \mu \mathrm{m}$ using a vibratome (Microm HM 650V, Thermo Scientific). After slicing, the tissue was incubated for $30 \mathrm{~min}$ at $37^{\circ} \mathrm{C}$ in an extracellular medium containing (in $\mathrm{mM}$ ): $125 \mathrm{NaCl}, 2.5 \mathrm{KCl}, 1 \mathrm{MgCl}_{2}$, $2 \mathrm{CaCl}_{2}, 1.25 \mathrm{NaH}_{2} \mathrm{PO}_{4}, 0.4$ ascorbic acid, 3 myo-inositol, 2 Na-pyruvate, 25 D-glucose, $25 \mathrm{NaHCO}_{3}$ (pH 7.4 in carbogen). Afterward, slices were kept at room temperature until the time of recording.

\section{Slice Recordings}

For recording, slices were placed in the holding chamber of an upright microscope (BX-50, Olympus) and MNTB principal cells were visualized using a $40 \times$ (NA 0.8) water immersion objective. For recording of $\mathrm{Na}^{+}$currents, slices were incubated at room temperature, and perfused with a $\mathrm{Na}^{+}$-selective solution containing (in $\mathrm{mM}$ ): $2.5 \mathrm{KCl}, 1 \mathrm{MgCl}_{2}, 2 \mathrm{CaCl}_{2}, 3$ myoinositol, $2 \mathrm{Na}$ pyruvate, $125 \mathrm{TEACl}, 0.1$ 3,4 diaminopyridine, $25 \mathrm{NaHCO}_{3}, 25 \mathrm{D}$-glucose, 0.4 ascorbic acid ( $\mathrm{pH}$ 7.4). MNTB afferents were stimulated using a bipolar electrode (FHC Inc.) positioned at the midline or half-way between the midline and the MNTB. Whole cell voltage clamp recordings were performed using borosilicate glass pipettes (3-4 M $\Omega$ ) filled with (in $\mathrm{mM}$ ): $130 \mathrm{Cs}$-gluconate, $20 \mathrm{CsCl}, 5 \mathrm{Na}_{2}$-phosphocreatine, 4 MgATP, $0.3 \mathrm{Na}_{2} \mathrm{GTP}, 0.5$ EGTA, 10 HEPES, pH 7.2. Recordings were performed in whole cell voltage clamp configuration. Whole cell series resistance $(<8.5 \mathrm{M} \Omega)$ was compensated by $95-98 \%$ at a lag of $9 \mu \mathrm{s}$. Membrane potentials were corrected on-line for a liquid junction potential of $-13 \mathrm{mV}$. The voltage dependence of recovery from inactivation of sodium channels was tested with a paired-pulse protocol; two $3 \mathrm{~ms}$ steps to $-10 \mathrm{mV}$ were given at time intervals ranging from 0.2 to $600 \mathrm{~ms}$ from a holding potential of $-95,-65$, or $-60 \mathrm{mV}$.

Stimulus trains were delivered using Clampex 8.2 (MDS Analytical Technologies) protocols via a Digidata 1320A 16-bit A/D converter (MDS Analytical Technologies). Data was 
acquired using an Axopatch 200B amplifier (MDS Analytical Technologies), filtered at $2-10 \mathrm{kHz}$ with a low pass, four-pole Bessel filter, and sampled at 66.67 or $83.33 \mathrm{kHz}$ with a Digidata 1320A.

\section{Analysis of in Vivo Recordings}

Researchers were blinded to genotype until analysis was completed. The analysis was performed using custom procedures written in the NeuroMatic environment (version 2.00, kindly provided by Dr. J. Rothman, University College London, London, United Kingdom; RRID:SCR_004186) within Igor Pro 6.2 (WaveMetrics, RRID:SCR_000325).

All in vivo recordings reported in this paper showed evidence for the presence of a prespike. Since it was hard to delineate the amplitude of juxtacellular EPSP (eEPSP) from the eAP in many recordings, we used the maximum rate of rise of eEPSP as a measure for the strength of transmission, as previously described (Wang et al., 2013).

To allow measuring the size of prespike at short intervals in juxtacellular recordings, the effects of the previous event on the baseline were subtracted using a custom template procedure. First, complex extracellular waveforms that were not immediately followed by another complex waveform within $2.5 \mathrm{~ms}$ were sorted into 4-6 subgroups based on their maximum downward rate and averaged following removal of outliers. For each complex waveform, the averaged waveform was found that had the best matching downward phase; this averaged waveform was aligned with the event at the point during the downward phase where the baseline was intersected (presumably corresponding with the peak of the intracellular AP; Lorteije et al., 2009), scaled, and subtracted from the event to allow a more accurate measurement of the prespike amplitude on the next event (Figures 8A,B).

The EPSP amplitudes or maximum rates of rise were fitted with a simple model for short-term plasticity (Varela et al., 1997), as described previously (Crins et al., 2011). In its simplest form, a single depression state parameter decreases at each event with a fraction called the depletion factor (comparable to the release probability of the terminal) and recovers continuously with a single time constant. Synaptic transmission is equal to the product of the depression state parameter and the transmission strength in the absence of shortterm plasticity.

We estimated the AP threshold of the MNTB neuron in two different ways. Firstly, because of the difficulty to separate EPSP and AP, we measured the point where the first derivative of EPSP reached its maximum, and took that point as the AP threshold. Secondly, we also measured AP threshold by analysis of subthreshold events in neurons with failures. A plot of the peak membrane potential reached during subthreshold EPSPs against event interval showed a similar distribution of peak membrane potentials at intervals $>10 \mathrm{~ms}$, but more positive potentials as the intervals decreased, indicating decreased excitability, due to refractoriness (Figure 5). We used the most positive peak potentials at long intervals as an alternative estimate for the AP threshold. This estimate correlated well with the inflection potential $(r=0.97)$, but was on average $5 \mathrm{mV}$ more negative.
Note that we use the term 'excitability' as any change that increases the probability that a given synaptic conductance may trigger an AP, including a change in recovery from spike depression.

\section{Analysis of Sodium Currents in Slice Recordings}

The amplitudes of $\mathrm{Na}^{+}$currents were measured as the difference between peak and local baseline using a custom-written Igor procedure. The start and end positions of the local baseline were defined by the user.

\section{Morphology of the Calyx}

Afferent fibers to the calyx of Held were electroporated with Alexa Fluor 594-labeled dextrans (10,000 MW; Invitrogen, Cat\# D22913) at the midline in vivo in young adult mice (P30-P60) as described previously (Rodríguez-Contreras et al., 2008). One hour later, the animal was perfused and the brainstem was sliced into $40-\mu \mathrm{m}$-thick sections. Postsynaptic cells were stained with SYTOX Blue (1:1000; Invitrogen, Cat\# S11348).

A laser scanning confocal microscope (LSM 700; Zeiss) equipped with krypton-argon and helium-neon lasers was used to acquire high resolution $z$-stack images (0.5 $\mu \mathrm{m}$ steps; $63 \mathrm{X}$ oil immersion objective, NA 1.4) of randomly selected calyces of Held using optimized laser power, detector gain, and pinhole diameter settings. Signal-to-noise ratio was defined as previously described (Di Guilmi et al., 2014), and was at least 20. The number of swellings per calyx was counted on 3D-rendered (Volocity 4.2; Improvision, RRID: SCR_002668) images of calyces with adjusted contrast and brightness. The surface area and volume of the calyces were measured using the region-ofinterest function in Volocity, images of calyx terminals were binary thresholded using the built-in thresholding function of ImageJ 1.46 (isodata algorithm, RRID: SCR_003070).

\section{Immunohistochemistry}

After recording, mice were perfused transcardially and the brains were post-fixed with $4 \%$ paraformaldehyde in sodium phosphate buffer (PB) for $1 \mathrm{~h}$. After sinking in 10 and $30 \%$ sucrose (in $0.1 \mathrm{M} \mathrm{PB}$ ), coronal brainstem sections were cut on a sliding microtome (SM2000R; Leica Microsystems, Rijswijk, Netherlands) at a thickness of $40 \mu \mathrm{m}$. The brain sections were washed in Tris-buffered saline (TBS; pH 7.6) and were incubated for $1 \mathrm{~h}$ in blocking buffer containing $10 \%$ horse serum, $0.5 \%$ Triton X-100 in TBS. Subsequently, sections were incubated for $48-72 \mathrm{~h}$ in TBS containing primary antibodies, $2 \%$ horse serum, and $0.4 \%$ Triton. The following primary antibodies were used: mouse anti-ankyrin-G (NeuroMab, clone N106/36, Cat\# 75-146, RRID: AB_10673030; 1:1000 dilution), rabbit anti-Nav1.6 (Alomone labs, Cat\# ASC009, RRID: $A B \_2040202$; 1:100), guinea pig anti-VGLUT1 and 2 (Chemicon; 1:1000 dilution), rabbit anti-CASPR2 (Chemicon; 1:500). After incubation with primary antibodies, the sections were washed in TBS, and transferred into TBS containing $2 \%$ horse serum, $0.4 \%$ Triton $\mathrm{X}-100$, and 1:1000 dilutions of 
Alexa Fluor-594 labeled anti-mouse antibody (Invitrogen, Cat\# A-11005, RRID: $\left.A B \_141372\right)$, Alexa Fluor-633 labeled antirabbit antibody (Invitrogen, Cat\# A-21070, RRID: $A B \_2535731$ ), Alexa-488 labeled anti-guinea pig antibody (Invitrogen, Cat\# A-11073 RRID: $\left.A B \_142018\right)$. After a 2-h incubation at room temperature, the sections were again washed with TBS and $0.1 \mathrm{M} \mathrm{PB}$, followed by a 1-min staining with Sytox Blue (Invitrogen). Afterward, sections were washed with $0.1 \mathrm{M}$ $\mathrm{PB}$, mounted on cover slides and covered with Vectashield (Vector Laboratories, Peterborough, United Kingdom). Confocal images were acquired with an LSM 700 (Carl Zeiss, Sliedrecht, Netherlands).

For UBE3A immunohistochemistry, procedures were the same as above, except brains were embedded in a sucrose/gelatin mixture (10 and $12 \%$, respectively), incubation with primary antibody (mouse anti-E6AP, E8655 Sigma-Aldrich; 1:2,000, RRID: $\left.A B \_261956\right)$ was overnight, the secondary antibody (anti-mouse HRP, P0447 Dako; 1:200, RRID:AB_2617137) was detected by $3,3^{\prime}$-diaminobenzidine (DAB) as the chromogen, and $\mathrm{DAB}$ sections were analyzed and photographed using a Leica DM-RB microscope and a Leica DFC450 digital camera.

\section{Quantification of AIS Length}

Ankyrin-G immunosignal, which appeared to be evenly distributed throughout the AIS, was used in the quantification of AIS length. The immunosignal of Vglut1 and CASPR2 were used to identify the calyx of Held and nodes of Ranvier, respectively. High resolution $z$-stack confocal images were acquired as described above. The $z$-stack images of initial segments were thresholded using the 3D Hysteresis Thresholding ImageJ plugin. The lower threshold was determined by thresholding a single plane in the middle of the image stacks with the IsoData algorithm. The higher threshold was set at twice the lower threshold. Thresholded image stacks were then loaded in Volocity, and the length of initial segment was quantified by applying the skeletal length measurement to the 3D-rendered structures.

\section{Western Blot Analysis}

To collect tissue for Western blot analysis, brain tissue was dissected from adult mice and immediately frozen in liquid nitrogen. The lysates were prepared by homogenization in lysis buffer (10 mM Tris-HCL pH 6.8, 2.5\% SDS) supplemented with protease inhibitor cocktail (P8340, Sigma-Aldrich). After centrifugation ( $6000 \mathrm{rpm}$ for $5 \mathrm{~min}$ ) supernatants were collected and concentration was measured using the Pierce BCA protein assay kit (\#23225, ThermoFisher Scientific). A total of $20 \mu \mathrm{g}$ of each sample was loaded on the gel and a wet transfer was performed. The blotted nitrocellulose membrane was probed with antibodies directed against E6AP (E8655 Sigma-Aldrich; 1:1,000, RRID: $\left.A B \_261956\right)$ and Actin (MAB1501R Millipore; 1:20,000, RRID: $\left.A B \_2223041\right)$. A fluorophore-conjugated secondary Goat anti-mouse antibody (Westburg, IRDye 800CW; 1:15,000, RRID: $\left.A B \_2687825\right)$ was used and the protein was detected using Li-cor Odyssey Scanner system. Quantification was done using Odyssey 3.0 software (Li-cor Biosciences).
Number of animals used for quantification was 4 for each genotype.

\section{Statistical Analysis}

Data is presented as the mean \pm standard error of the mean (SEM). Statistical significance of differences between means was assessed using Student's $t$-test or Mann-Whitney $U$ test if data were not normally distributed. No correction for multiple testing was applied.

\section{RESULTS}

\section{Generation of Ube3a Mice}

Ube $3 a$ mutants carrying an E113X nonsense mutation in exon 5 were generated and crossed into $\mathrm{C} 57 \mathrm{BL} / 6 \mathrm{~J}$ as described in the Section "Materials and Methods" and Figure 1A. Immunohistochemistry showed a strong reduction of UBE3A protein throughout the brain (Figure 1B). This reduction was quantified by Western blot analysis of the cortex, hippocampus and brainstem, showing that mice with a maternally inherited Ube $3 a^{E 113 X}$ mutation had a $80-90 \%$ reduction of UBE3A protein (Figure 1C). This reduction is similar to the commonly used Ube3a ${ }^{\text {Exon3 }}$ mice (Jiang et al., 1998).

\section{Normal Hearing Thresholds in Ube3a Mice}

We used ABR to compare hearing thresholds in $U b e 3 a^{E 113 X}$ mice and WT controls in the C57BL/6J background. At the age where we tested the mice, thresholds at $32 \mathrm{kHz}$ were not yet elevated, and WT controls had thresholds that were similar to published values (Zheng et al., 1999). Hearing thresholds of Ube $3 a^{E 113 X}$ mice ( $n=11$ animals) and WT controls ( $n=6$ animals) were similar (Figure 2).

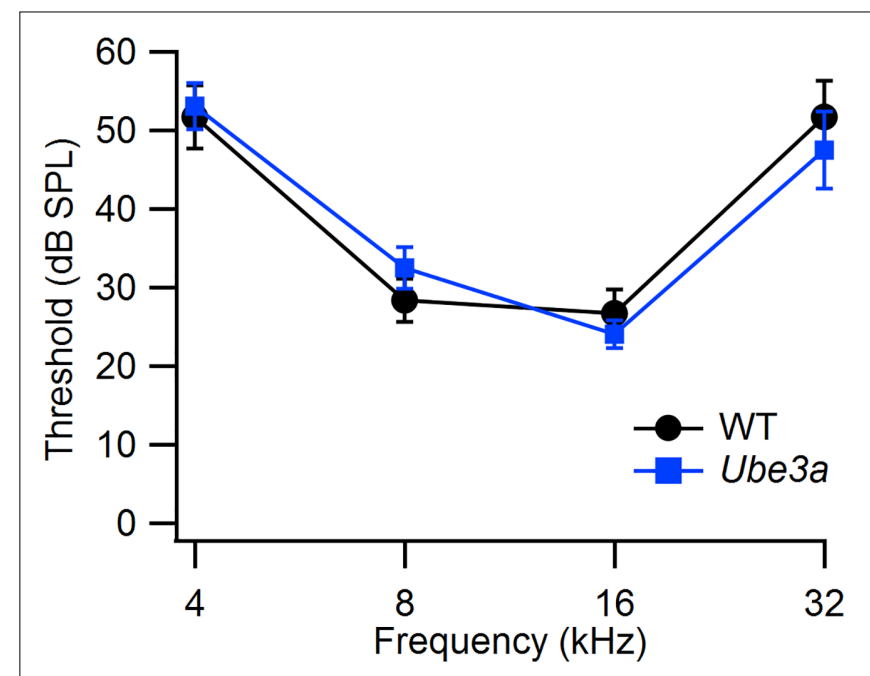

FIGURE 2 | Similar hearing thresholds in WT and Ube3a mice. Comparison of the hearing thresholds between WT $(n=6)$ and Ube3a ${ }^{E 113 X}$ mice $(n=11)$ at four different frequencies. 


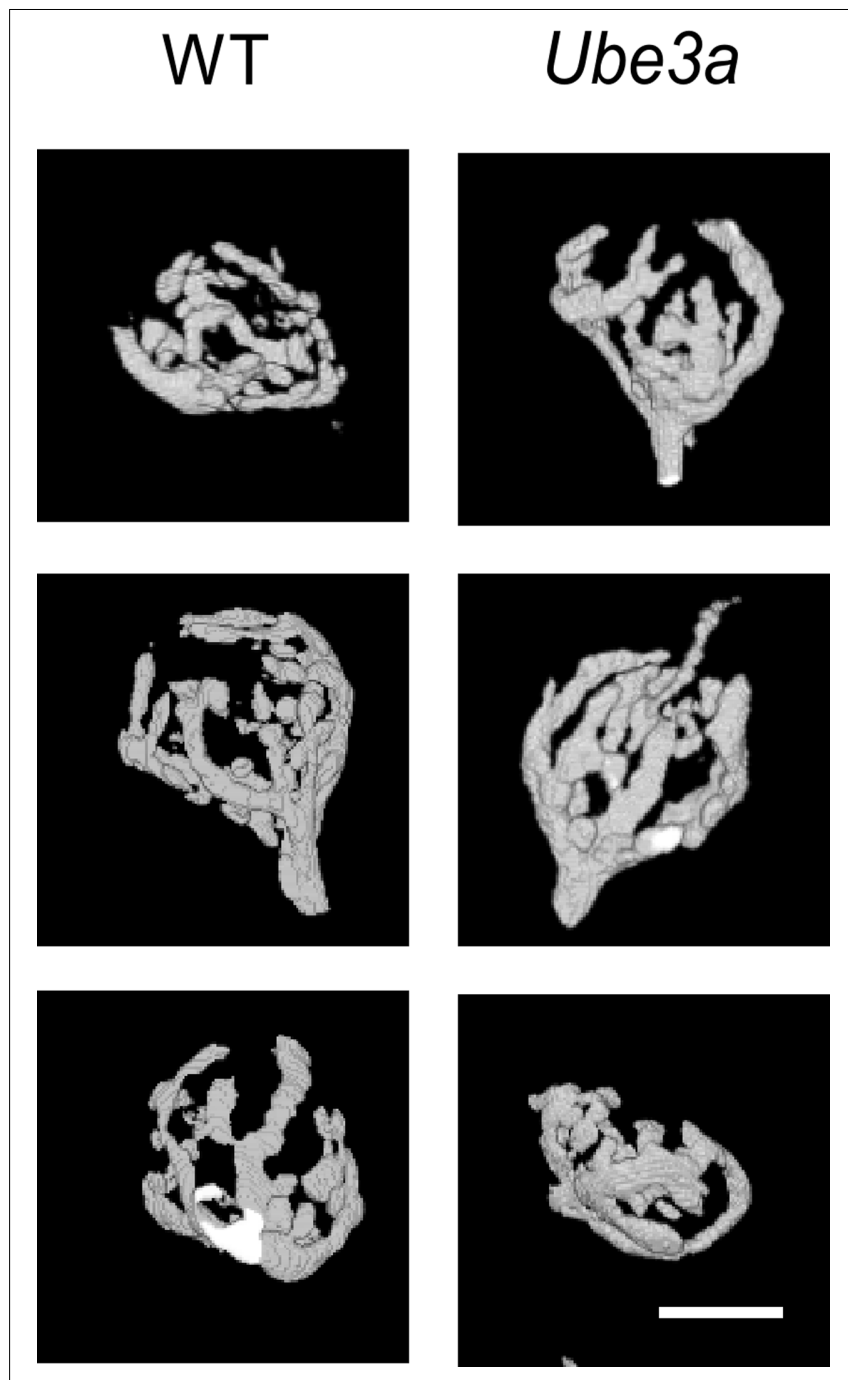

FIGURE 3 | Comparison of morphology of calyx of Held from WT and Ube3a mice. Images of 3D-rendered representative calyces from WT (left column) and Ube $3 a^{E 113 X}$ mice (right column). Scale bar, $10 \mu \mathrm{m}$.

\section{Calyx of Held Has Normal Morphology in Ube3a Mice}

We compared the morphology of the calyx of Held terminal, which forms a relay synapse in the auditory brainstem, in Ube $3 a^{E 113 X}$ mice and wild-type (WT) littermates in the C57BL/6J background. The mature calyx of Held synapse has a complex structure with fingers containing numerous stalks and swellings (Morest, 1968). Afferent fibers of globular bushy cells (GBCs) were fluorescently labeled in vivo by electroporation in P30P60 animals, followed by confocal imaging of fixed brainstem slices (Figure 3). Z-stack confocal images were reconstructed in $3 \mathrm{D}$, and the number of boutons, surface area as well as the volume of the calyx was compared between Ube $3 a^{E 113 X}$ and WT. Fifteen Ube $3 a^{E 113 X}$ calyces (from five animals) and six WT calyces (from two animals) were reconstructed. All calyces had swellings originating from thin necks of third order
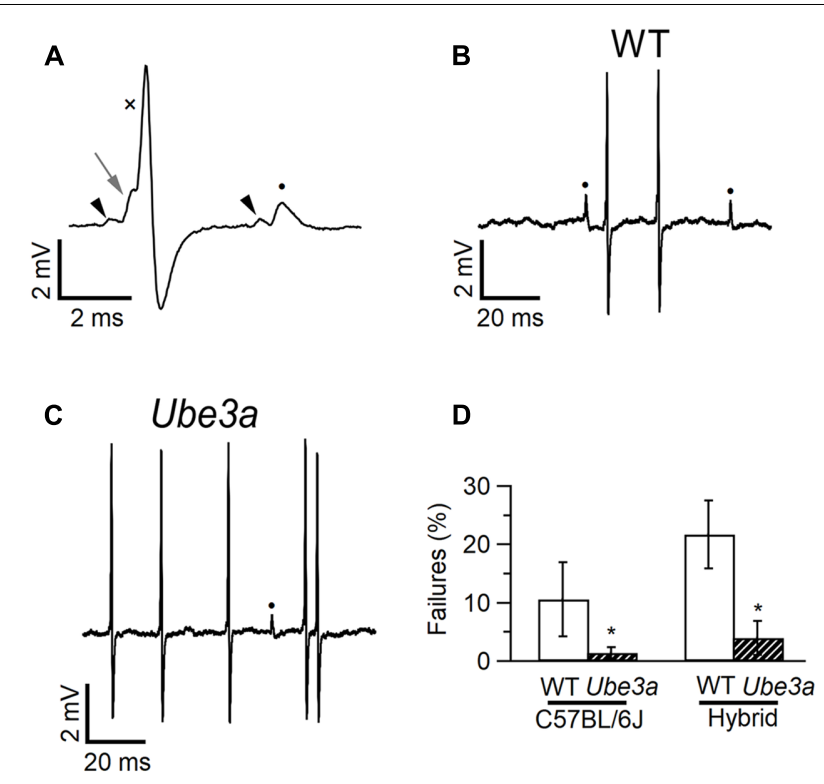

FIGURE 4 | Fewer failures in MNTB neurons of Ube3a mice. (A) Two complex waveforms from principal neuron of a C57BL/6J WT mouse recorded in the juxtacellular configuration, showing a suprathreshold eEPSP (left gray arrow), which triggers an eAP (cross), and a subthreshold eEPSP (closed circle), which is not followed by an eAP. Both eEPSPs are preceded by a prespike (black arrow heads). (B) Same recording as $\mathbf{A}$, illustrating the presence of both subthreshold (filled circles) and suprathreshold events. (C) Similar as B, but from a Ube3aE113X mouse. (D) Comparison of spontaneous failure percentages of WT and Ube3a mice from two genetic backgrounds. *Indicates $p<0.05$ (Mann-Whitney $U$ test).

branches. We classified the calyces into three groups according to a previous morphological study (Grande and Wang, 2011): terminals in group I had $<6$ swellings, group II had 6-14, and group III had at least 15 swellings. In WT calyces, 3 of 6 calyces belonged in group II and the remainder in group III. In the KI, 8 of 15 calyces belonged in group II and the remainder in group III. Terminals with 6 or less swellings were not observed (Di Guilmi et al., 2014; Wang et al., 2015). Surface area $\left(443 \pm 47 \mu^{2}\right.$ in Ube3a vs. $454 \pm 53 \mu \mathrm{m}^{2}$ in WT; $p=0.89)$ and volume of calyx terminals $\left(1225 \pm 80 \mu \mathrm{m}^{3}\right.$ in $\mathrm{KI}$ vs. $1286 \pm 100 \mu \mathrm{m}^{3}$ in WT; $\left.p=0.67\right)$ were also similar between Ube $3 a^{E 113 X}$ mutant and WT calyces. We therefore conclude that the gross morphology of WT and Ube $3 a^{E 113 X}$ terminals was similar.

\section{Decreased Failure Rate in Ube3a Mice}

Epilepsy is a common feature of AS (Williams et al., 2006), and audiogenic seizures can be observed in an AS mouse model, but its presence is strongly dependent on genetic background (Van Woerden et al., 2007; Silva-Santos et al., 2015). To investigate changes in excitability and synaptic transmission in the auditory pathway in AS mice, we studied the in vivo synaptic activity of the calyx of Held synapse. The recordings were performed in the juxtacellular (loose-patch) configuration, in two independent Ube $3 a$ lines: the traditionally used Ube3a knock-out line in which exon 3 is deleted (Ube3a $a^{\text {Exon3 }}$ mice; Jiang et al., 1998) 

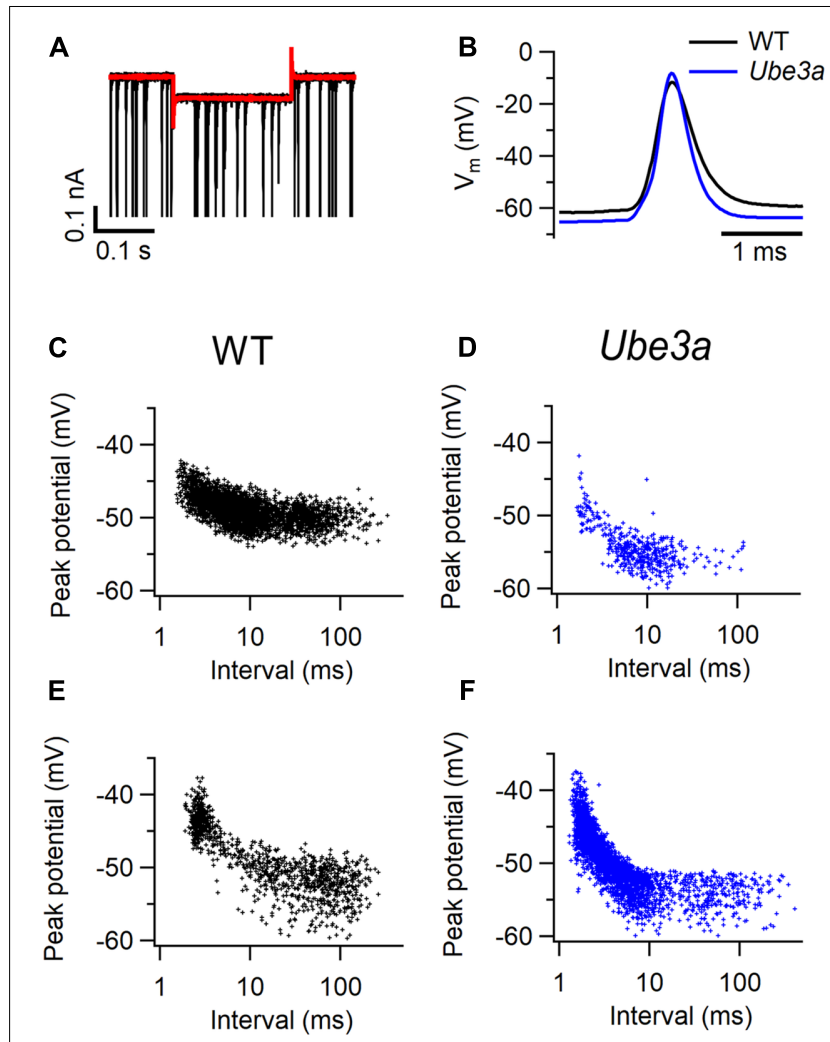

FIGURE 5 | Intrinsic properties of MNTB neurons in Ube3a mice. (A) Representative superimposed action potentials (APs) from a C57BL/6J WT (black) and a Ube3a ${ }^{E 113 X}$ (blue) mouse, recorded in vivo in the whole-cell configuration. (B) Superimposed average trace (red), and the overlay of 5 original traces with spontaneous activity (black) during a $0.2 \mathrm{~s}, 10 \mathrm{mV}$ hyperpolarizing step from a holding potential of $-70 \mathrm{mV}$. (C-F) Relation between most positive potential reached in subthreshold EPSPs and inter-EPSP interval. Data is from two cells from C57BL/6J WT animals (C,E) and two cells from Ube3a ${ }^{E 113 X}$ animals (D,F).

in the hybrid F1 129/Sv-C57BL/6 background, and a novel Ube3a mutant in the C57BL/6 background harboring a E113X nonsense mutation in exon 5 (Ube $3 a^{E 113 X}$ mice). Both lines result in the loss of maternal UBE3A protein expression. The identification of single-unit recordings from principal neurons was based on their characteristic complex waveform (Guinan and $\mathrm{Li}, 1990)$. The complex waveforms consist of a small prespike and a larger, brief, positive deflection, which have previously been shown to originate from the calyx of Held and the principal cell, respectively (Lorteije et al., 2009). The good signal-to-noise ratio obtained with the juxtacellular (loosepatch) configuration allowed a clear discrimination between suprathreshold and subthreshold synaptic events (Figure 4A). The average spontaneous firing frequency was on average higher in Ube $3 a^{E x o n 3}$ mice in the hybrid $129 \mathrm{~S} 2 / \mathrm{Sv}-\mathrm{C} 57 \mathrm{BL} / 6$ background compared to Ube $3 a^{E 113 X}$ mice in the C57BL/6 background, but these frequencies were similar compared to their respective wild-type littermates (Table 1). The percentage of subthreshold events was greatly reduced (Figures $4 \mathrm{~B}, \mathrm{C}$ ). Wild-type mice in the C57BL/6J background showed postsynaptic failures in 10
TABLE 2 | Comparison of spontaneous firing, complex waveforms and intrinsic properties between WT and Ube3a ${ }^{113 X}$ mice.

\begin{tabular}{lccc}
\hline Parameters (whole-cell) & WT & Ube3aE113X & p-value \\
\hline Spontaneous failures (\%) & $13.5 \pm 7.6$ & $8.5 \pm 5.2$ & 0.59 \\
Spontaneous frequency (Hz) & $19 \pm 5$ & $26 \pm 3$ & 0.34 \\
Membrane resistance $(\mathrm{M} \Omega$ ) & $72.4 \pm 7.1$ & $93.6 \pm 7.3$ & 0.06 \\
Resting potential (mV) & $-61.8 \pm 2.4$ & $-67.2 \pm 1.5$ & 0.04 \\
AP amplitude & $47.9 \pm 2.4$ & $54.9 \pm 2.3$ & 0.05 \\
AP dVdt (V/s) & $152.3 \pm 14.8$ & $203.1 \pm 18.1$ & 0.05 \\
AP half width (ms) & $0.55 \pm 0.04$ & $0.44 \pm 0.03$ & 0.03 \\
Inflection point (mV) & $-57.6 \pm 2.0$ & $-60.2 \pm 1.4$ & 0.15 \\
Estimated threshold (mV) & $-52.2 \pm 2.9$ & $-55.0 \pm 2.4$ & 0.6 \\
\hline
\end{tabular}

Data is from in vivo whole-cell recordings from WT ( $n=8$ cells from seven animals) and Ube3a ( $n=9$ cells from seven animals). Statistical differences of the mean were assessed by t-test.

of 14 cells, and the average failure rate was $10.6 \pm 6.4 \%$. In contrast, in Ube $3 a^{E 113 X}$ mice in the C57BL/6J background only 4 out of 15 cells exhibited failures, and the average percentage of failures was only $1.4 \pm 1.0 \%$ ( $p=0.026$, MannWhitney $U$ test). Moreover, we observed a similar significant decrease in spontaneous failures in the Ube $3 a^{E x o n 3}$ mice in the hybrid 129S2/Sv-C57BL/6J background (Figure 4D and Table 1). Given that we observed this phenomenon in two independent Ube3a mutants with different genetic backgrounds, these results strongly point toward a critical role of UBE3A in controlling the strength of synaptic transmission.

We next examined the time course and shape of the complex extracellular waveforms (Table 1). AS mice exhibited significantly shorter eEPSP-eAP delays compared to WT littermates, whereas prespike-eEPSP delays were not statistically different. This phenotype was again observed in both mutants and genetic backgrounds.

To further investigate the intrinsic properties of MNTB principal neurons in Ube $3 a$ mice, whole-cell recordings were performed in vivo in Ube3a $a^{E 113 X}$ mice with the C57BL/6J background. In voltage clamp mode, a $-10 \mathrm{mV}$ hyperpolarizing step was applied (Figure 5A), and series resistance as well as membrane resistance were calculated (see "Materials and Methods"). Ube $3 a$ mice and WT littermates showed similar membrane resistances (Table 2). Little or no evidence for the presence of the non-selective, hyperpolarization-activated cation channel $I_{\mathrm{h}}$ was observed during the hyperpolarizing voltage step, suggesting that in vivo little $I_{\mathrm{h}}$ is active around the resting membrane potential in both Ube3a mice and WT littermates (Figure 5A). Spontaneous and evoked AP waveforms were recorded in current clamp mode. MNTB neurons of the Ube3a mutants exhibited a more hyperpolarized resting membrane potential than wild-type mice (Table 2). Moreover, AP amplitude and its maximum rate of rise were larger in Ube3a mutants compared to their wild-type littermates, whereas width at halfmaximum was shorter in Ube3a mutants (Figure 5B). Since previous studies showed lower AP threshold in pyramidal neurons of AS mice (Kaphzan et al., 2011), we measured AP threshold of the MNTB neuron in two different ways, as detailed in the Methods. There was no significant difference in the 


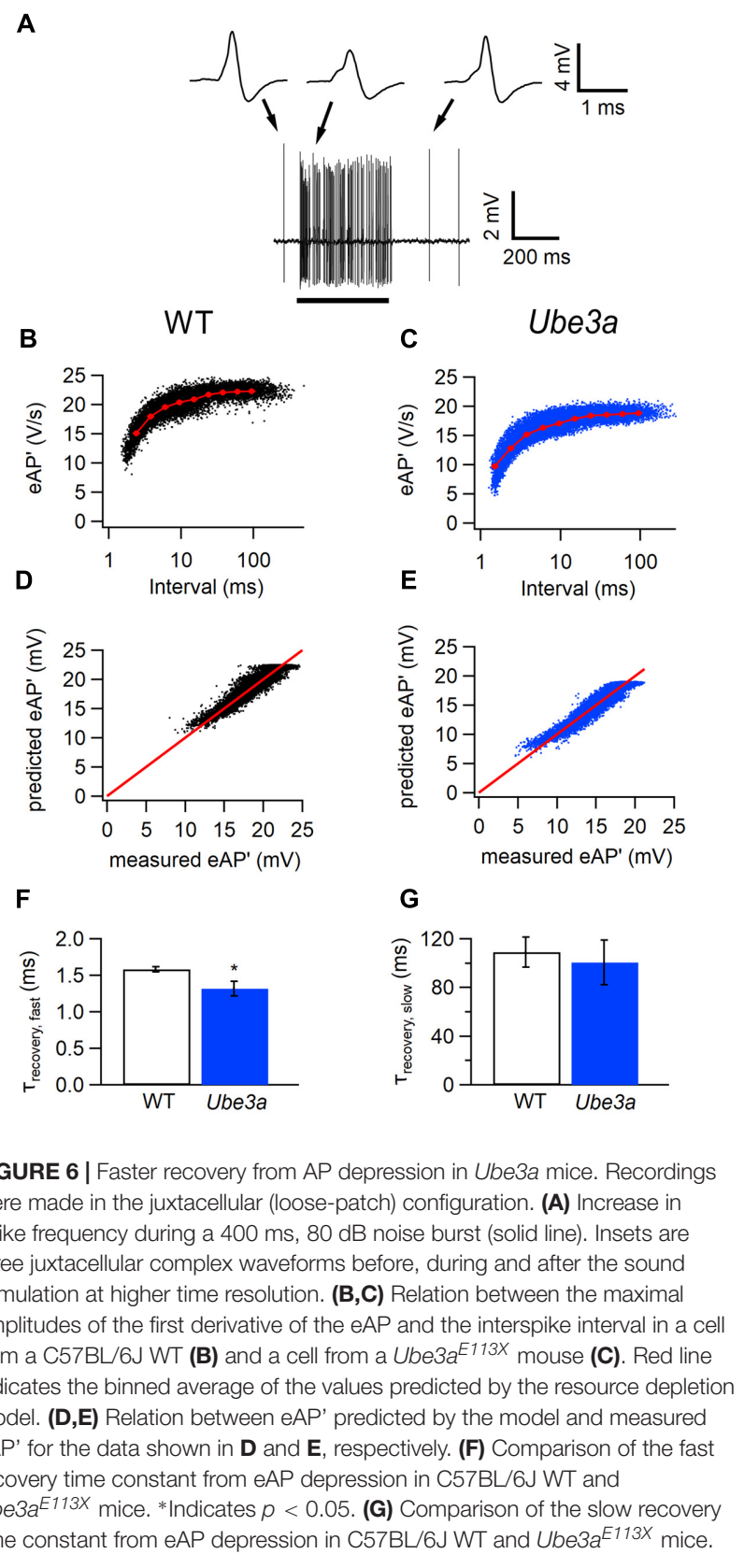

threshold potential between Ube3a mice and their wild-type littermates measured based on the inflection point between EPSP and AP or based on failure analysis (Figures 5C-F and Table 2).

\section{Ube3a Mice Show Enhanced Recovery from Postsynaptic AP Depression}

To further investigate the difference in APs between the Ube $3 a$ mutants and WT mice, we estimated recovery time constants from AP depression. Presentation of a $400 \mathrm{~ms}, 80 \mathrm{~dB}$ noise burst elicited a clear increase in firing rate. The amplitude of juxtacellularly recorded eAP was attenuated during auditory stimulation, to which presumably sodium channel inactivation
A
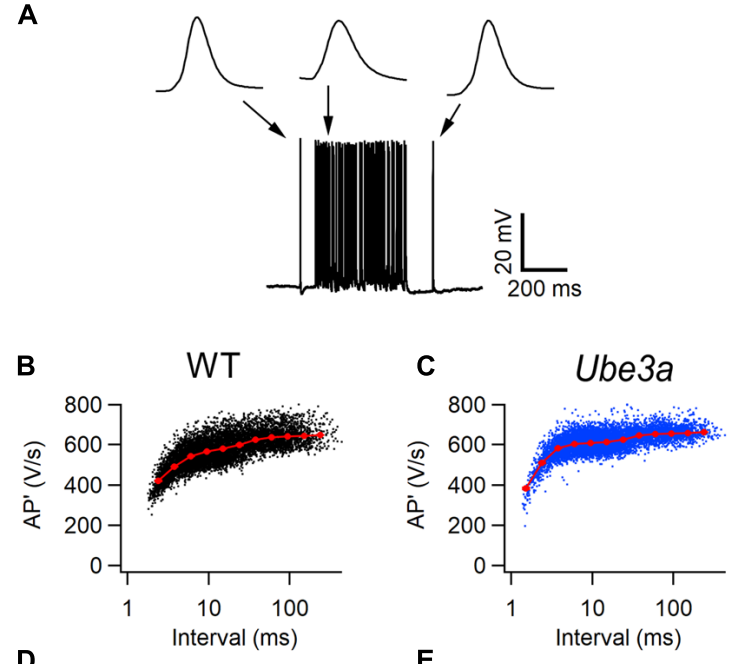

D

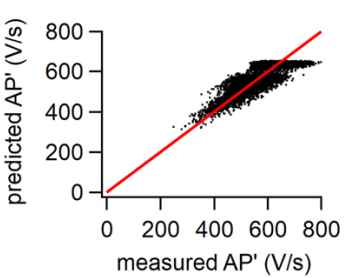

$\mathbf{F}$

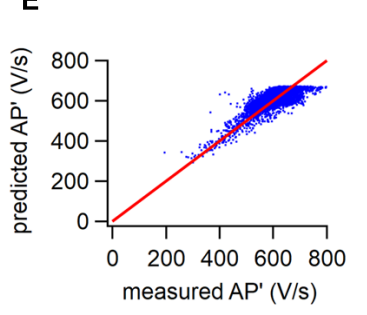

G
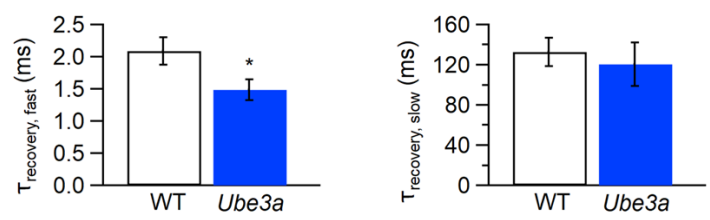

FIGURE 7 | Faster recovery from AP depression in whole-cell configuration in Ube3a mice. (A) Increase in spike frequency during a $400 \mathrm{~ms}, 80 \mathrm{~dB}$ noise burst in a whole-cell recording. Insets are three principal neuron APs before, during and after the sound stimulation, respectively. $(\mathbf{B}, \mathbf{C})$ Relation between the maximal amplitudes of the first derivative of the AP (AP') and the interspike interval from a whole-cell recording (black) from a WT animal (B) and a Ube3a mouse (C). Red line indicates the binned average of the values predicted by the resource depletion model. (D,E) Relation between AP' predicted by the model and measured in the whole-cell recording for the data shown in $\mathbf{B}$ and C, respectively. (F) Comparison of the fast recovery time constant from eAP depression between WT and Ube3a mice. *Indicates $p<0.05$.

(G) Comparison of the slow recovery time constant from eAP depression between WT and Ube3a mice.

made a large contribution, and gradually recovered to the original level after the stimulus (Figure 6A). To study the recovery of postsynaptic excitability, we measured the maximum of the first derivative of the extracellularly recorded AP (eAP') at different inter-AP intervals (Figures 6B,C). The relation between the size of the eAP' and inter-AP interval was fitted with a simple resource depletion model (Varela et al., 1997), as previously described (Lorteije et al., 2009). The recovery of eAP' could be well described by the sum of a fast and a slow time constant (Figures 6D-G). The fast time constant of recovery from eAP' depression was significantly smaller in Ube $3 a^{E 113 X}$ mice than 
in C57BL/6J WT ( $\tau_{\text {Ube3a }}: 1.32 \pm 0.10 \mathrm{~ms}, n=14$ cells from four animals vs. $\tau_{\mathrm{WT}}: 1.58 \pm 0.04 \mathrm{~ms}, n=15$ cells from three animals; $p=0.02$; Figures $6 \mathbf{B}-\mathbf{F}$ ). The slow time constant of recovery did not differ between $U b e 3 a^{E 113 X}$ and C57BL/6J WT $\left(\tau_{\text {Ube3a }}: 100 \pm 18 \mathrm{~ms}\right.$ vs. $\tau_{\mathrm{WT}}: 109 \pm 12 \mathrm{~ms} ; p=0.7$; Figure 6G). The model fit explained a large part of the variance in eAP' amplitudes, both in Ube $3 a^{E 113 X}$ mutants and in C57BL/6J WT animals ( $80.6 \pm 2.7 \%$ and $80.7 \pm 2.4 \%$, respectively).

To test whether postsynaptic APs recorded in the whole-cell configuration also recovered more rapidly from depression in Ube $3 a$ mice, we plotted the maximum of the first derivatives of AP' against the inter-AP intervals (Figures 7A-C). A fit with the resource depletion model showed that the fast time constant for recovery from spike depression was clearly smaller in the Ube $3 a^{E 113 X}$ mice $(1.48 \pm 0.16 \mathrm{~ms}, n=8$ vs $2.09 \pm 0.22 \mathrm{~ms}$, $n=6$, respectively; $p=0.04$; Figures $7 \mathbf{B}-\mathbf{G})$, similar to results obtained in juxtacellular recordings. The faster recovery from AP depression suggests that the kinetics of sodium channel recovery from inactivation are altered in Ube3a mice, although a contribution of changes in potassium channel deactivation cannot be excluded.

\section{Ube3a Mice Show Enhanced Recovery from Presynaptic AP Depression}

Next, we tested whether the presynaptic AP were also affected. Information about the calyceal AP can be obtained by measuring the amplitude of the prespikes. When the interval between events is short, the prespike of the next event often falls in the downward deflection of the previous one, making quantification of prespike amplitude difficult. Since the pre- and postsynaptic contribution to the complex extracellular waveform are in principle independent voltage sources, we addressed this problem by subtracting a scaled, averaged waveform from the previous event, as detailed in the Methods and illustrated in Figures 8A,B. Only cells with prespikes larger than $0.3 \mathrm{mV}$ were included in this analysis. We plotted the amplitudes of the prespikes against the inter-spike interval, and fitted their relation with a simple model (Varela et al., 1997). The relation between prespike amplitude and the inter-spike interval could be adequately described with a single recovery time constant. Similar to the postsynaptic AP, the prespike of Ube3a mice also recovered significantly faster than WT $(2.53 \pm 0.28 \mathrm{~ms}, n=4$ cells from four animals vs $3.88 \pm 0.45 \mathrm{~ms}, n=4$ cells from four animals; $p=0.043$; Figures 8C-E). The presynaptic $\mathrm{Na}^{+}$currents depend on the $\mathrm{Na}_{\mathrm{V}} 1.6$ subunit at the pre-calyceal axonal heminode (Leão et al., 2005; Berret et al., 2016; Sierksma and Borst, 2017), and our results suggest possible changes in the recovery from inactivation of this channel.

\section{No Changes in Recovery from Inactivation in Postsynaptic Sodium Channels from Ube3a Mice}

To test whether the faster recovery from postsynaptic AP depression in the Ube3a mice were caused by a faster recovery from inactivation of voltage-dependent sodium channels, we made whole-cell voltage recordings in slices while
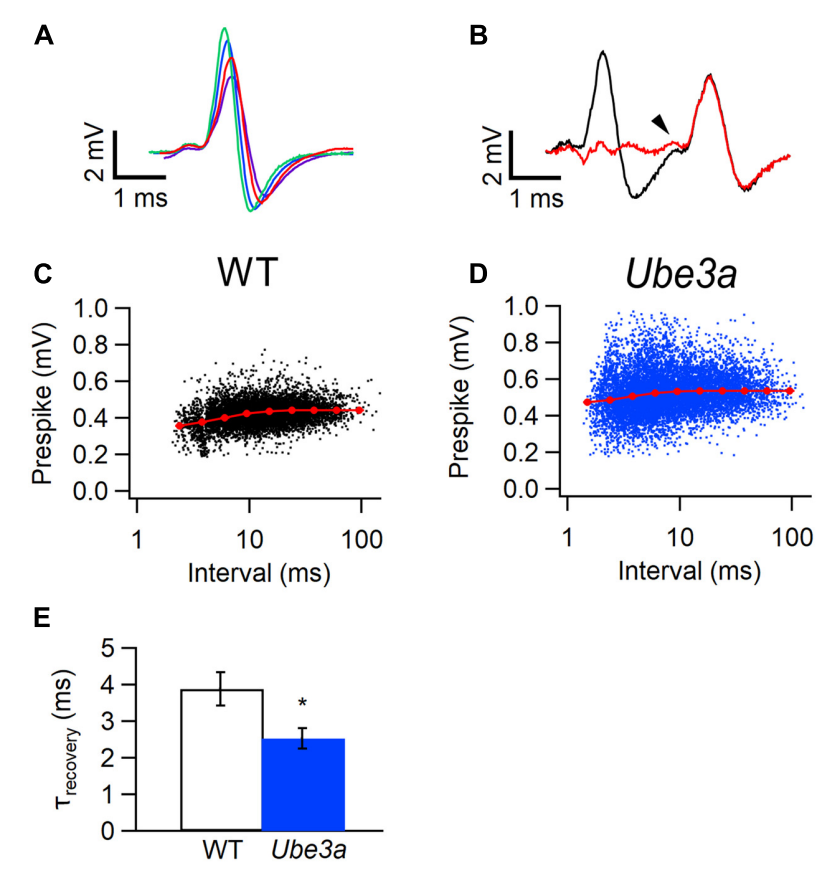

FIGURE 8 | Faster recovery from prespike depression in Ube3a mice. Recordings were made in the juxtacellular configuration. (A) Four templates used for subtraction; each template was made by averaging complex waveforms with similar amplitudes from a recording from a WT mouse (hybrid background). (B) Baseline before (black) and after (red) subtraction of a matching template from the first of the two complex waveforms from the same recording as in $\mathrm{A}$. The prespike is indicated by an arrow head. (C,D) Relation between the amplitudes of the juxtacellularly recorded prespikes and inter-EPSP intervals from a WT (C, hybrid background) or a Ube3a ${ }^{\text {Exon3 }}$ animal (D). Red symbols indicate binned averages of the values predicted by the resource depletion model. (E) Comparison of the recovery time constant from pre-AP depression between WT and Ube3a animals (in both groups three mice with hybrid background and one C57BL/6J mouse). *Indicates $p<0.05$.

pharmacologically isolating the sodium channels. We studied recovery from inactivation with two steps to $-10 \mathrm{mV}$ with variable interval at holding potentials of $-95,-65$, or $-60 \mathrm{mV}$ (Figures 9A,B). The first step to $-10 \mathrm{mV}$ induced a rapid and almost full inactivation. Recovery from inactivation could generally be well described by a single exponential function, whose time constant depended strongly on the potential in between the steps (Figures 9C,D). Time constants were in the same range as the fast time constant from AP depression measured in vivo, although spatial clamp problems and a difference in temperature (voltage clamp studies were done at room temperature), present some uncertainties. The voltage dependence of sodium channel recovery in WT and Ube3a mice was very similar (Figures $\mathbf{9 E}-\mathbf{H}$ ), suggesting that the observed difference in AP recovery was not caused by a change in the kinetics of the sodium channels.

\section{Increased AIS Length of Principal Neurons in the Ube3a Mutant}

Our observations indicate that the Ube3a mouse is a gain of function mutant for synaptic transmission in the calyx of Held 


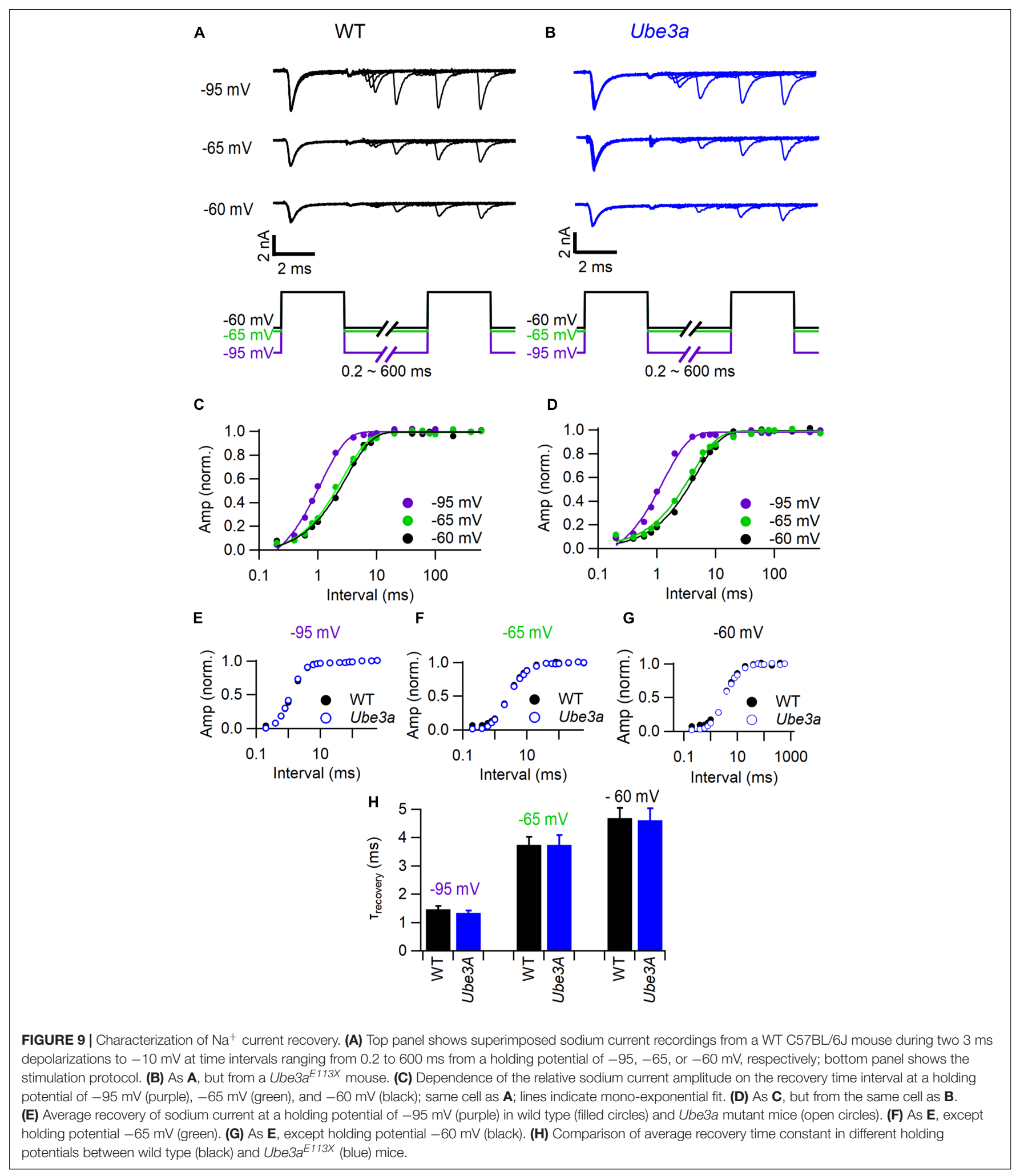

synapse. Voltage-gated sodium channel Nav1.6 plays a critical role in controlling neuronal excitability and AP generation (Kuba et al., 2014). Its expression level is significantly higher and the length of the AIS is significantly increased in the CA1 and CA3 hippocampal subregions of Ube3a mice (Kaphzan et al., 2011). We therefore measured the AIS length of principal neurons in the MNTB using the AIS-organizing protein ankyrin-G as a marker (Kim et al., 2013). Contactin-associated protein 2 


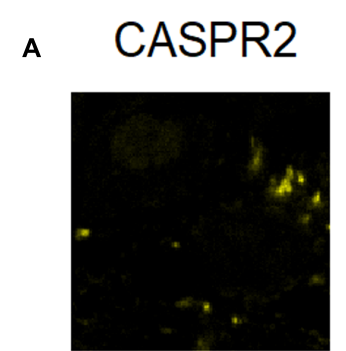

\section{ank-G}
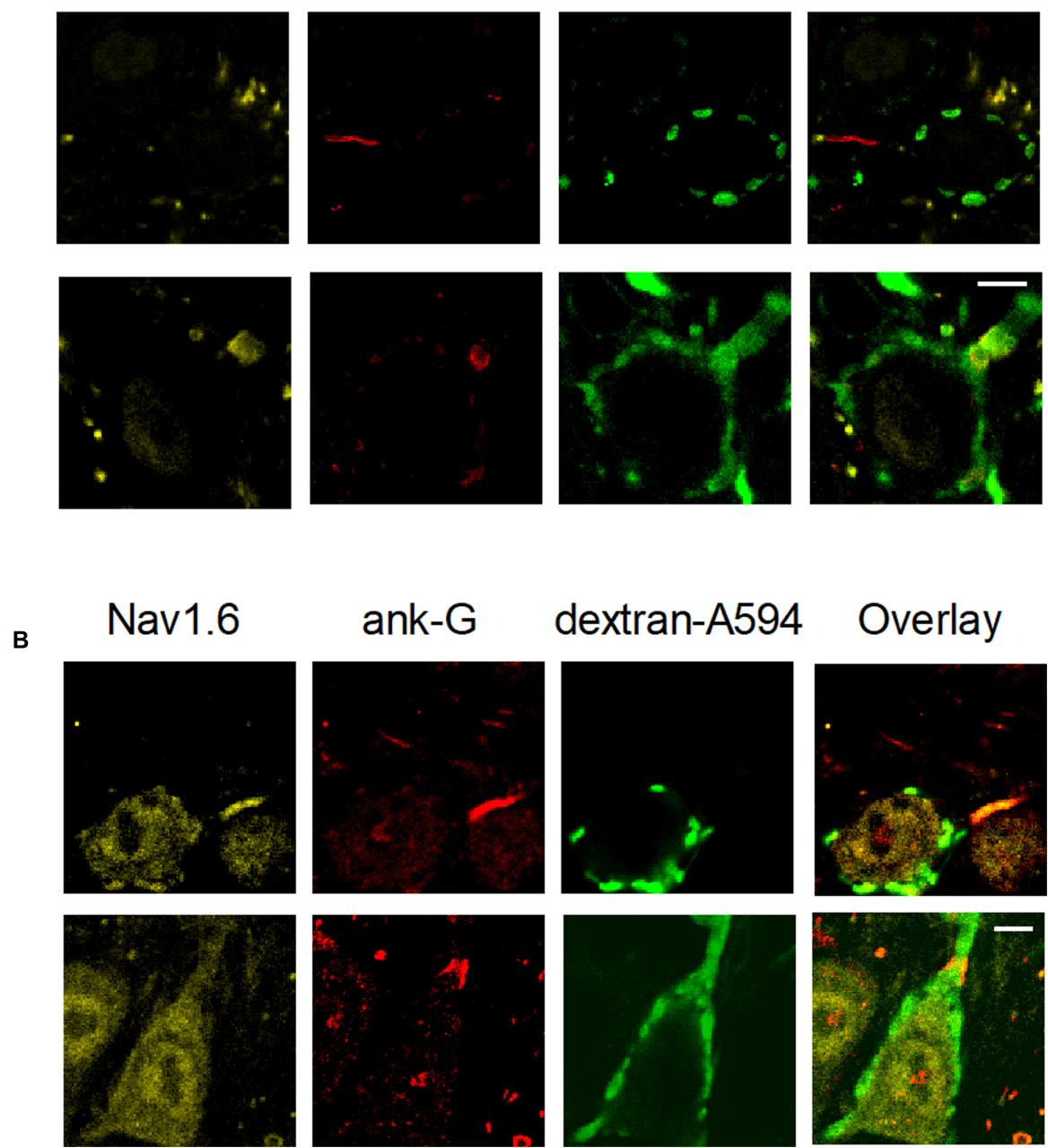

dextran-A594
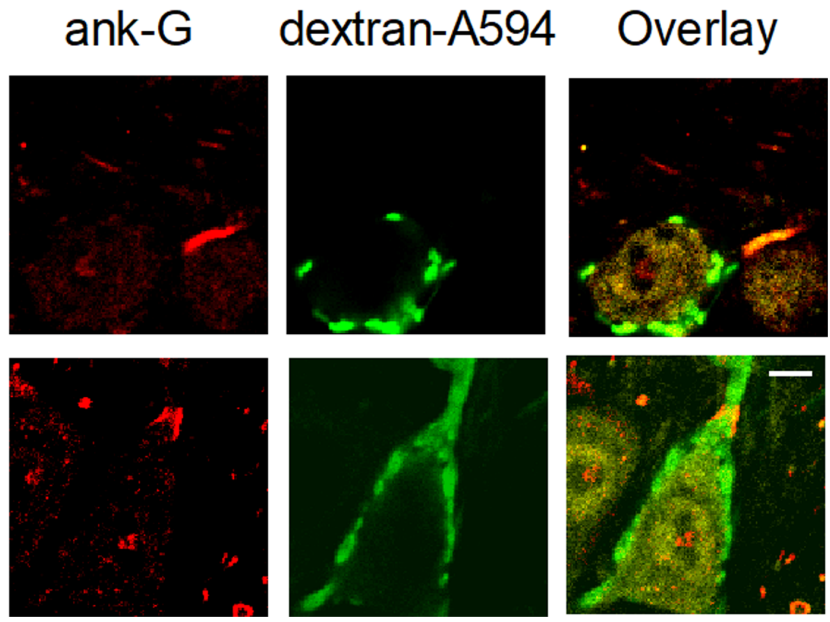

FIGURE 10 | Structural differences between heminodes and axon initial segments (AIS) in the MNTB. Calyces were filled with dextran-A594 via midline electroporation. (A) A filled calyx stained with anti-CASPR2 and anti-ankyrin-G antibody, as well as an overlay of these images. (B) A filled calyx with staining of anti-Nav1.6 and anti-ankyrin-G antibody, as well as an overlay of these images. Calibration bars $5 \mu \mathrm{m}$.

(CASPR2), a neurexin protein localized at the juxtaparanodes of myelinated axons (Poliak et al., 1999), was also stained to help discriminate between pre- and postsynaptic axonal structures. AIS immunolabeling of ankyrin-G revealed three types of structures: short structures that were flanked on both sides by CASPR2-positive staining, identifying them as nodes of Ranvier, a thin, bar-like structure that was followed by CASPR2 staining at one end, and a much thicker and shorter structure that always partially co-localized with CASPR2 on one end (Figure 10A). To distinguish between the calyceal heminode and the principal cell axons, afferent fibers of GBCs were fluorescently labeled via in vivo electroporation, before immunostaining with the combination of anti-ankyrin-G and anti-CASPR2/anti- $\mathrm{Na}_{\mathrm{v}}$ 1.6. The thick structures always appeared to be part of the fluorescently labeled fibers before the start of the calyx terminal (Figure 10), which indicates these were heminodes (Ford et al., 2015; Xu et al., 2016). In the 758 axons we have measured, the thin, bar-like structures never coincided with fluorescently labeled fibers, and they were always followed by CASPR2 staining at the distal end to the principal neuron, suggesting that the thin bar-like structure is the AIS, which is immediately followed by myelination of principal cell axon. These results are in agreement with the previous finding that the calyceal axon is clearly thicker than the principal cell axon (Forsythe, 1994; Leão et al., 2005). There was also intensive labeling by anti- $\mathrm{Na}_{\mathrm{v}} 1.6$ at the region of AIS (Figure 10B), suggesting a significant colocalization of sodium channels and ankyrin-G at the initial segment. Similar to the morphological difference between calyceal heminode and principal cell axon, the presynaptic nodes of Ranvier also appeared thicker than the postsynaptic ones (Figure 11A).

Having established that we can reliably identify the AIS, we next quantified the length of the AIS of MNTB cells from both 


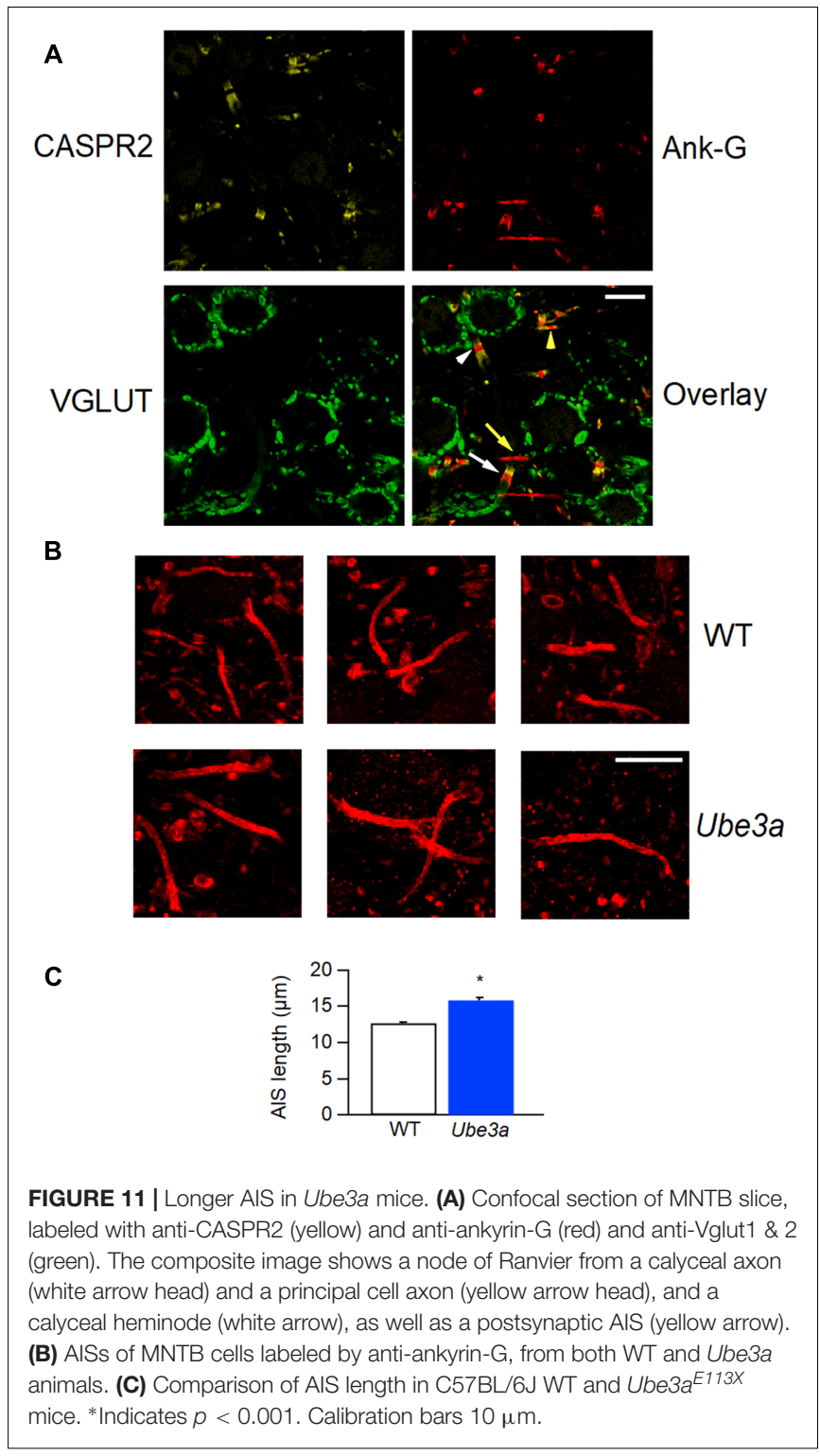

C57BL/6J WT and Ube $3 a^{E 113 X}$ mice. The bar-like ankyrin-Gpositive structures from Ube $3 a$ mice were on average longer than in their WT littermates $(12.7 \pm 0.2 \mu \mathrm{m}$ in WT, from 520 AIS of 6 mice vs. $15.9 \pm 0.3 \mu \mathrm{m}$ in Ube3a from 238 AIS of 3 mice; $p<0.001$; Figures 11B,C). Assuming a similar density of sodium channels at the AIS of WT and Ube3a mice, together with the co-staining results shown in Figure 11B, the increased length of the AIS suggests that an increased number of sodium channels are expressed in principal neurons of Ube3a mice.

\section{Ube3a Mice Show Diminished Short-term Depression in Vivo}

Since the threshold of AP did not change, the lower failure rates of spontaneous synaptic events of AS mice might have resulted from a difference in the calyceal input. For this
A

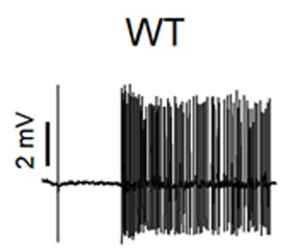

C
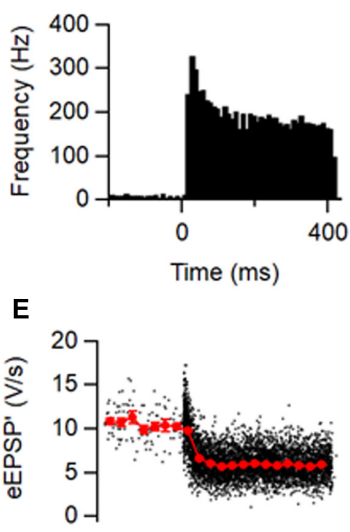

G

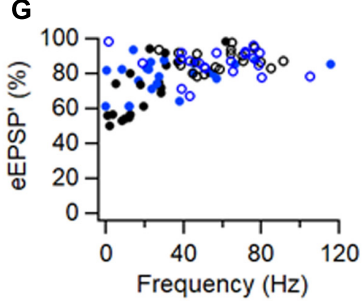

B

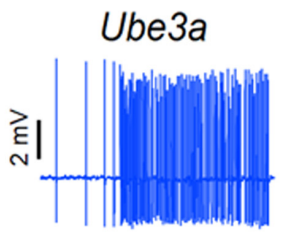

D

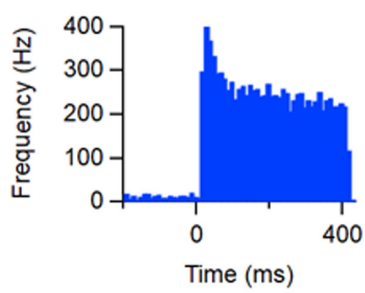

F

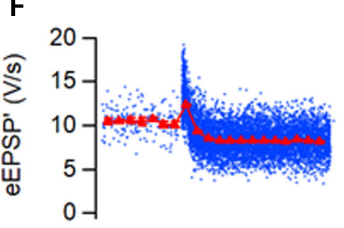

H 100 마 $\begin{aligned} & \text { WT } \\ & \text { Ube3a }\end{aligned}$

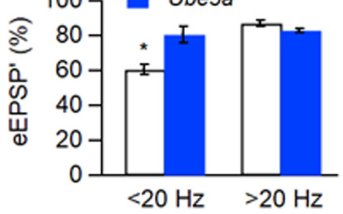

FIGURE 12 | Smaller sound-evoked STD in Ube3a mice. (A) Increase in frequency during a $400 \mathrm{~ms}, 80 \mathrm{~dB}$ noise burst. (C) Peristimulus time histogram showing primary-like response to sound. (E) Amplitudes of eEPSP' Closed circles indicate time-binned averages. Bin size $30 \mathrm{~ms}$. (A,C,E) are from the same WT juxtacellular recording from a C57BL/6J mouse. (B,D,F) are similar with $(\mathbf{A}, \mathbf{C}, \mathbf{E})$, respectively, except the recording was from a unit in a Ube3aE113X mouse. (G) Relation between eEPSP' amplitude and spontaneous firing frequency. WT and Ube3a animals are indicated in black and blue, respectively. Closed circles and open circles represent cells from C57BL/6J background and 129S2/Sv-C57BL/6J background, respectively. (H) Comparison of relative eEPSP' amplitudes during the last $50 \mathrm{~ms}$ of auditory stimulation of cells with a spontaneous frequency $<20 \mathrm{~Hz}$ and $>20 \mathrm{~Hz}$, respectively. ${ }^{*}$ Indicates $p<0.05$.

reason, we studied STD of the first derivative of the EPSP (EPSP') evoked by sound stimulation. Spontaneous firing in C57BL/6J WT animals averaged $21 \pm 4 \mathrm{~Hz}(n=20$; range $1-62 \mathrm{~Hz}$ ). During auditory stimulation, it reached a maximum frequency of $311 \pm 11 \mathrm{~Hz}$ within the first $10 \mathrm{~ms}$ and decayed to $155 \pm 7 \mathrm{~Hz}$ during the last $50 \mathrm{~ms}$ of the tone (Figures 12A,C). Spontaneous firing frequencies in the Ube $3 a^{E 113 X}$ animals averaged $32 \pm 6 \mathrm{~Hz}(n=23$; range $0.05-116 \mathrm{~Hz}$ ). Ube3a mutants had similar maximum firing frequencies during the first $10 \mathrm{~ms}$ of auditory stimulation, and steady-state frequencies during the last $50 \mathrm{~ms}$ as WT animals (Figures 12B,D). 


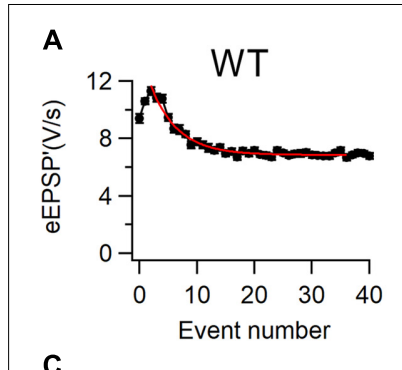

C

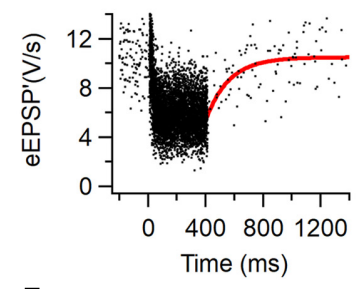

E

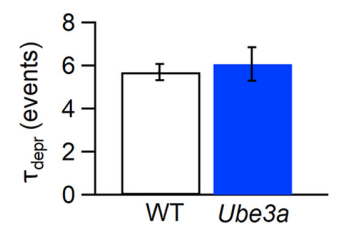

B

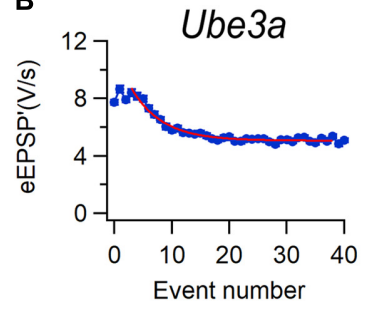

D

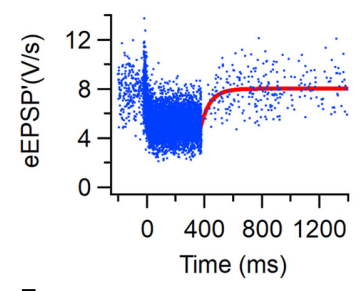

F

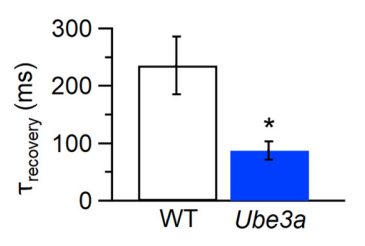

FIGURE 13 | Faster kinetics of recovery from STD in Ube3a mice.

(A) Amplitude of eEPSP' against sound-evoked event number from recording of C57BL/6J WT cell. Error bars indicate SEM. Solid line is fit with single exponential function. (C) Sound-evoked eEPSP' in a WT animal. Solid line is fit of recovery from sound-evoked STD with single exponential function with time constant $215 \mathrm{~ms}$. (B,D) Both are similar with $\mathbf{A}$ and $\mathbf{C}$, respectively, except that the recording was from a Ube3a mouse $31 \mathrm{~ms}$. (E) Comparison of results of fit of depression time course as shown in $A$ and $B$ for WT and Ube3a animal. (F) Comparison of recovery time constants as shown in $\mathbf{C}$ and D for WT and Ube3a. A and $\mathbf{C}$ are from the same WT recording; $\mathbf{B}$ and $\mathbf{D}$ are from the same unit of a Ube3a mouse. *Indicates $p<0.01$.

We used the maximum of the first derivative of the extracellular EPSP (eEPSP') or the second derivative of wholecell EPSP (EPSP") as a measure for the strength of synaptic transmission, as described previously (Wang et al., 2013). During sound presentation, the average eEPSP' in both WT and Ube $3 a^{E 113 X}$ mice often decreased to a lower level, indicating that the high firing frequencies induced STD (Figures 12E-G). Overall, the Ube $3 a^{E 113 X}$ mutants showed less STD than the WT controls ( $80 \pm 2 \%$ of control, $n=23$ vs. $71 \pm 3 \%, n=20 ; p=0.04)$. As previously observed (Wang et al., 2013, 2015), the amount of STD in WT animals depended strongly on spontaneous firing frequencies (Figure 12H), presumably because cells with a high spontaneous firing frequency are tonically depressed. In C57BL/6J WT mice, cells with a spontaneous frequency $<20 \mathrm{~Hz}$ showed large STD $(61 \pm 3 \%$ of control, $n=11$ cells from five animals). In contrast, STD in Ube3a cells exhibited no dependency on spontaneous firing (Figure $\mathbf{1 2} \mathbf{H}$ ). The Ube $3 a^{E 113 X}$ cells with a spontaneous frequency $<20 \mathrm{~Hz}$ showed little STD (78 $\pm 6 \%$ of control, $n=8$ cells from seven animals; $p=0.01$ vs. WT).

\section{EPSPs from Ube3a Mice Show Faster Recovery from Depression}

To further investigate the difference in STD between $U b e 3 a^{E 113 X}$ and WT mice, we estimated the time course of synaptic depression and recovery in both juxtacellular and whole-cell recordings. To quantify how many events it took for the synapse to reach steady-state depression, the relation between the eEPSP' and its event number was plotted (Figures 13A,B,E). The decay of the amplitudes could be well fitted by a single exponential function, as previously described (Wang et al., 2013). During auditory stimulation, Ube $3 a^{E 113 X}$ and C57BL/6J WT took a similar number of events to reach steady state $\left(\tau_{U b e 3 a}: 6.1 \pm 0.7\right.$ events, $n=22$ cells, including eight whole-cell recordings vs. $\tau_{\mathrm{WT}}: 5.7 \pm 0.4$ events, $n=20$ cells, including six whole-cell recordings; $p=0.67$ ). For the estimation of the time course for recovery from STD, eEPSP' (or EPSP' in the case of whole-cell recordings) was plotted against time, and fitted with a single exponential function (Figures 13C,D,F). In contrast to the lack of an obvious difference in the time course of the onset of depression (Figures 13A,B,E), the recovery from depression was overall much faster in Ube3a $a^{E 113 X}$ than C57BL/6J WT cells $\left(\tau_{U b e 3 a}\right.$ : $88 \pm 14 \mathrm{~ms}, n=21$ cells, including eight whole-cell recordings vs. $\tau_{\mathrm{WT}}: 235 \pm 51 \mathrm{~ms}, n=19$ cells, including six whole-cell recordings; $p=0.006$ ). To confirm these results, we also fitted the data by a short-term plasticity model (Varela et al., 1997), which takes the firing frequency and firing pattern into account. The predicted time constant of recovery from depression was again faster in Ube3a $a^{113 X}$ than in WT cells ( $\tau_{\text {Ube } 3 a}: 141 \pm 16 \mathrm{~ms}, n=20$ cells including 8 whole-cell recordings vs. $\tau_{\mathrm{WT}}: 193 \pm 22 \mathrm{~ms}$, $n=16$ cells including 6 whole-cell recordings; $p=0.06$ ), which is in agreement with the direct fits of the recovery after the sound bursts.

\section{DISCUSSION}

Here we show that maternal loss of Ube3a leads to enhanced synaptic transmission at the calyx of Held synapse in vivo. The Ube3a mutant MNTB neurons showed decreased failure rate compared to the wild type. Both pre- and postsynaptic APs recovered faster from depression. These phenotypes were replicated in two independent Ube3a mutants in two different genetic backgrounds. In the in vivo whole-cell recording of Ube $3 a^{E 113 X}$ mice in the C57BL/6J background, the mutant neurons exhibited altered intrinsic properties, including elevated AP amplitude and decreased AP half width, as well as a more hyperpolarized resting membrane potential. We also observed an increased AIS length in the Ube $3 a^{E 113 X}$ mice in the C57BL/6J background, which might be related to the larger AP. In addition, reduced STD and faster recovery from STD were observed. Taken together, these in vivo results imply a critical role of UBE3A in controlling synaptic transmission and excitability at excitatory synapses.

\section{Synaptic vs. Network Changes}

Previous studies of Ube3a mice have focused mainly on cortical and hippocampal neurons (Kaphzan et al., 2011; Wallace et al., 
2012, 2017). These neurons are innervated by a heterogeneous population of synapses, making it more difficult to distinguish the impact of UBE3A on the many different inputs. In the case of the calyx of Held synapse, synaptic transmission can be studied in relative isolation. Because of the similarity in hearing thresholds and in spontaneous and sound-evoked frequencies in WT and Ube3a mice, it is unlikely that the enhanced neurotransmission is caused by upstream effects or an excitatory/inhibitory imbalance within the MNTB, also taking into account that in vivo evidence suggests that the direct contribution of inhibitory inputs at the mouse calyx of Held synapse is limited (Lorteije and Borst, 2011). Apparently, the observed increased reliability of excitatory transmission was not sufficient to lead to a substantial increase in spontaneous firing frequency in the MNTB.

\section{Increased Resistance to Spike Depression}

Two main differences in the function of the calyx of Held synapse were observed between $U b e 3 A$ mice and their wildtype littermates. We observed an increased resistance to STD, presumably a presynaptic change in the young-adult calyx of Held synapse, and an increased excitability, probably both preand postsynaptically. The two gain-of-function phenotypes are probably unrelated, as the effect on STD acts at about a 100 times slower timescale than some of the effects on excitability. We will first discuss the effects on excitability.

Postsynaptically, we observed a more negative membrane potential, fewer failures, a larger AP and smaller AP half width, and a longer AIS. Both pre- and postsynaptically, we observed faster recovery from AP depression. Several of these findings are most likely related to postsynaptic sodium channels. During high frequency stimulation, AP amplitudes will depress. We used the amplitudes of the juxtacellularly recorded prespike and postspike, which provide a measure for the maximum rate of rise of the pre- and the postsynaptic AP, respectively (Lorteije et al., 2009), to compare AP depression between Ube3a and WT. In this study, we observed that APs from Ube3a principal MNTB cells recovered faster from depression than in WT. In addition, evidence for faster recovery of calyceal APs in vivo was obtained. Recovery of the rate of rise of the AP amplitude can be expected to depend foremost on the recovery of sodium channels from the inactivated state, which is both a time- and a voltage-dependent process (Ming and Wang, 2003).

The recovery from presynaptic spike depression could be described by a single exponential function, in agreement with the mono-exponential recovery from inactivation of sodium channels in calyces (Leão et al., 2005) or hippocampal mossy fiber boutons (Engel and Jonas, 2005). A direct comparison of the kinetics of recovery of the sodium channels and of the spike depression shows that the spikes recovered somewhat more slowly, which may be related to the strong voltagedependence of the fast component of the recovery from sodium channel inactivation (Leão et al., 2005; Mathews, 2008). In addition, the small size of the prespikes, which at short interevent intervals could only be measured following a template subtraction procedure, makes the determination of the exact kinetics a challenging task.

We found that recovery from postsynaptic spike depression could be well described by the sum of two exponential functions, which was in agreement with our earlier results (Lorteije et al., 2009). The sodium channels of principal neurons also recover biphasically, at similar kinetics as the recovery from AP depression (Leão et al., 2005), suggesting - similar to the presynaptic situation - a prominent role for the recovery from sodium channel inactivation in recovery from AP depression. Somatic sodium channel density is low in the MNTB principal neurons (Leão et al., 2005, 2008), suggesting changes in the axonal domain. Postsynaptic measurements were somatic, whereas the axonal and somatic AP can differ substantially (Scott et al., 2007; Yu et al., 2008). The rate of rise of the somatic AP is influenced by the backpropagation from the AIS. Moreover, the backpropagating AP will interact with the large, axosomatic calyceal input. These considerations make inferences about the mechanisms underlying these observed changes somewhat speculative, especially with regard to possible changes during multiple APs.

The voltage clamp recordings from principal neurons in MNTB slices did not provide any evidence for a change in the voltage-dependent recovery from sodium channel inactivation in the Ube3a mice, even though we cannot exclude that small effects were missed, since the voltage clamp in the whole cell recordings was suboptimal, recordings were performed at room temperature, and the slow component previously observed by Leão et al. (2005) was not observed by us. Interestingly, the kinetics of the recovery from inactivation of sodium channels or MNTB principal neurons were shown to be different in deaf mice, but the difference was in the slow component of recovery (Leão et al., 2006), which was not altered in the Ube3a mice.

The most likely mechanism underlying the faster recovery from AP depression in the Ube3a mice is related to the more negative resting potential, which based on our voltage clamp recordings (Figure 9) and previous results (Leão et al., 2005; Mathews, 2008) should speed up recovery from sodium channel inactivation considerably. However, we cannot exclude that the observed changes in AP recovery were mediated by potassium channels, especially since we observed shorter AP half widths in the Ube $3 a$ principal neurons. The calyx of Held synapse contains a multitude of different potassium channels (Johnston et al., 2010), and a subset of these show rapid facilitation (Yang et al., 2014), which could contribute to spike depression. A concomitant switch in the $\mathrm{K}$ channel composition of the AIS contributes to the increased excitability observed in the chick nucleus laminaris following cochlear ablation (Kuba et al., 2015).

\section{Increase in AIS Length}

We saw about a $25 \%$ increase in AIS length of the principal neurons, somewhat larger than the increase observed previously in the hippocampus of Ube3a mice (Kaphzan et al., 2011). It is not known whether the observed increase in AIS length was accompanied by a change in its location, but in general, 
changes in AIS length, with corresponding changes in total sodium conductance, are more effective in regulating neuronal excitability than changes in location (Gulledge and Bravo, 2016). An increase in AIS length by itself may lead to shorter interspike intervals, a larger maximal rate of rise and more negative AP threshold (Kuba and Ohmori, 2009; Baalman et al., 2013). A longer AIS (with the resulting larger $\mathrm{Na}$ conductance) can help to overcome the electrical load of the soma and dendrites (Kuba et al., 2006; Baranauskas et al., 2013; Gulledge and Bravo, 2016; Hamada et al., 2016). The impact that a change in AIS length will have on neuronal excitability will also depend on changes in its localization and ion channel composition (Kuba et al., 2006, 2015; Baranauskas et al., 2013; Evans et al., 2015; Gulledge and Bravo, 2016; Hamada et al., 2016). A larger AIS may move spike initiation further away from the soma, making it less susceptible for the depolarization induced by calyceal EPSPs at the soma (Kuba et al., 2006; Scott et al., 2014). Even though some of the signal transduction mechanisms underlying AIS plasticity are beginning to be identified (Trunova et al., 2011; Galiano et al., 2012; Evans et al., 2013, 2015, 2017), how UBE3A contributes to the regulation of its length remains to be addressed.

Our results are largely consistent with a previous in vitro study showing a more negative resting membrane potential, larger AP amplitude and increased maximal rate of rise of the APs in CA1 pyramidal neurons of Ube3a mice (Kaphzan et al., 2011), but differ from a recent in vivo study in layer $2 / 3$ pyramidal neurons of the visual cortex, in which increased intrinsic excitability in Ube3a mice was found to be mainly due to an increased membrane resistance (Wallace et al., 2017). The hippocampal and cortical pyramidal neurons are more similar to each other than to MNTB neurons, which are fast, glycinergic neurons that serve mostly a relay function. Why the differences in excitability observed in the present study are more similar to the changes observed in hippocampal neurons than in neocortical neurons is presently not clear.

\section{Homeostatic Plasticity or Gain-of-Function?}

Evidence was presented that the more negative resting potential in hippocampal neurons likely result from increased expression of the $\alpha 1$ subunit of the $\mathrm{Na} / \mathrm{K}$-ATPase, and the changes in the AP from increased expression of Nav1.6 (Jiang et al., 1998; Kaphzan et al., 2011, 2013), and similar results have been obtained in the Drosophila neuromuscular junction (Valdez et al., 2015; Hope et al., 2017). The Nav1.6 subunit can be expected to be the dominant type of sodium channel in the young-adult MNTB (Leão et al., 2005). To explain the results in the hippocampus, it was suggested that the increase of the AIS length of Ube $3 a$ model mice might reflect a homeostatic response to a reduction in neuronal excitability, driven by the increase of $\alpha 1$-NaKA expression (Kaphzan et al., 2011, 2013). It has been well established that the AIS is a dynamic structure, and that the expression of sodium channels can depend on input activity (Grubb and Burrone, 2010; Kuba et al., 2010, 2014, 2015; Gutzmann et al., 2014; Nozari et al., 2017). It is remarkable that the increase in AIS length was associated with a more negative membrane potential, both at hippocampal CA1 pyramidal neurons (Kaphzan et al., 2011) and in the present study, since a chronic depolarization induces a rapid change in AIS length in cultured neurons (Evans et al., 2015). A homeostatic mechanism, as suggested as a compensatory mechanism for the changes in membrane potential observed in the hippocampus, seems insufficient to explain our results. A homeostatic mechanism would be expected to compensate for a decrease in excitability, thus keeping the reliability on average constant. However, we observed fewer postsynaptic failures in the Ube $3 a$ mutant mice in the absence of obvious changes in the input firing patterns to the principal neurons. This gain-of-function phenotype thus indicates that a simple homeostatic mechanism is insufficient to explain the observed results.

\section{Reduced STD}

During auditory stimulation, complex effects were observed on short-term synaptic depression (STD) of the EPSP. In the posthearing calyx of Held synapse, STD is presynaptic (Joshi and Wang, 2002; Taschenberger et al., 2002, 2005; Yamashita et al., 2003; Renden et al., 2005; Koike-Tani et al., 2008), even though it has not yet been confirmed that this also holds true for the young-adult mice that were studied here. In Ube3a animals, the sound-evoked EPSP showed less STD, and the depression level was largely independent of spontaneous activity, which contrasts with the Drosophila neuromuscular junction, where increased STD was observed (Valdez et al., 2015; Li et al., 2016). Most likely, the observed faster recovery from synaptic depression contributed to the reduction in STD we observed during tone presentation. We previously also observed faster recovery from STD in the Cacna1a ${ }^{S 218 L}$ mutant mouse model of Familial Hemiplegic Migraine (FHM; Di Guilmi et al., 2014). However, in the Cacnala $a^{S 218 L}$ knock-in mice increased STD was observed, probably due to increased release probability of the readily releasable pool owing to elevated basal $\left[\mathrm{Ca}^{2+}\right]_{i}$, indicating that there are essential differences in the presynaptic changes in the Cacna1 $a^{S 218 L}$ and the Ube3a mice.

\section{OUTLOOK}

Here we have provided the first in vivo characterization of the intrinsic membrane properties, synaptic transmission and AIS morphology of MNTB neurons in Ube3a mice. The enhanced synaptic transmission and elevated AP amplitude are likely to contribute to the hyperexcitability and increased seizure susceptibility in Ube $3 a$ mice and AS patients even though changes in GABAergic transmission are also an important factor in epileptogenesis (Judson et al., 2016). A homeostatic regulation of membrane excitability (Kaphzan et al., 2011, 2013) seems insufficient to explain the observed gain of function. How decreased UBE3A-mediated proteasomal degradation causes abnormalities in excitability and AIS structure deserves further study. The molecular mechanisms for the increased resistance to short-term synaptic depression in the calyx of Held synapse of Ube $3 a$ mice also remain to be identified. Identification of pre- and 
postsynaptic targets of UBE3A in the MNTB would be a helpful step toward these goals.

\section{AUTHOR CONTRIBUTIONS}

GvW and YE designed and generated the Ube $3 a^{E 113 X}$ mice. GvW did the Western blot and immunocytochemical analysis of these mice. TW and JB performed and analyzed electrophysiological experiments. TW did morphological analysis. JB wrote analysis software. TW and JB wrote the manuscript. All authors contributed to the planning of the experiments, commented on earlier versions of the manuscript, and approved the final version of the manuscript.

\section{REFERENCES}

Baalman, K. L., Cotton, R. J., Rasband, S. N., and Rasband, M. N. (2013). Blast wave exposure impairs memory and decreases axon initial segment length. J. Neurotrauma 30, 741-751. doi: 10.1089/neu.2012.2478

Baranauskas, G., David, Y., and Fleidervish, I. A. (2013). Spatial mismatch between the $\mathrm{Na}^{+}$flux and spike initiation in axon initial segment. Proc. Natl. Acad. Sci. U.S.A. 110, 4051-4056. doi: 10.1073/pnas. 1215125110

Berret, E., Kim, S. E., Lee, S. Y., Kushmerick, C., and Kim, J. H. (2016). Functional and structural properties of ion channels at the nerve terminal depends on compact myelin. J. Physiol. 594, 5593-5609. doi: 10.1113/JP272205

Borst, J. G. G., and Soria van Hoeve, J. (2012). The calyx of Held synapse: from model synapse to auditory relay. Annu. Rev. Physiol. 74, 199-224. doi: 10.1146/ annurev-physiol-020911-153236

Bruinsma, C. F., Schonewille, M., Gao, Z., Aronica, E. M. A., Judson, M. C., Philpot, B. D., et al. (2015). Dissociation of locomotor and cerebellar deficits in a murine Angelman syndrome model. J. Clin. Invest. 125, 4305-4315. doi: 10.1172/JCI83541

Buiting, K., Williams, C., and Horsthemke, B. (2016). Angelman syndrome insights into a rare neurogenetic disorder. Nat. Rev. Neurol. 12, 584-593. doi: 10.1038/nrneurol.2016.133

Burette, A. C., Judson, M. C., Burette, S., Phend, K. D., Philpot, B. D., and Weinberg, R. J. (2017). Subcellular organization of UBE3A in neurons. J. Comp. Neurol. 525, 233-251. doi: 10.1002/cne.24063

Crins, T. T. H., Rusu, S. I., Rodríguez-Contreras, A., and Borst, J. G. G. (2011). Developmental changes in short-term plasticity at the rat calyx of Held synapse. J. Neurosci. 31, 11706-11717. doi: 10.1523/JNEUROSCI.1995-11. 2011

Di Guilmi, M. N., Wang, T., Inchauspe, C. G., Forsythe, I. D., Ferrari, M. D., Van Den Maagdenberg, A. M. J. M., et al. (2014). Synaptic gain-of-function effects of mutant $\mathrm{Ca}_{v} 2.1$ channels in a mouse model of familial hemiplegic migraine are due to increased basal $\left[\mathrm{Ca}^{2+}\right]$ i. J. Neurosci. 34, 7047-7058. doi: 10.1523/jneurosci.2526-13.2014

Engel, D., and Jonas, P. (2005). Presynaptic action potential amplification by voltage-gated $\mathrm{Na}^{+}$channels in hippocampal mossy fiber boutons. Neuron 45, 405-417. doi: 10.1016/j.neuron.2004.12.048

Evans, M. D., Dumitrescu, A. S., Kruijssen, D. L. H., Taylor, S. E., and Grubb, M. S. (2015). Rapid modulation of axon initial segment length influences repetitive spike firing. Cell Rep. 13, 1233-1245. doi: 10.1016/j.celrep.2015. 09.066

Evans, M. D., Sammons, R. P., Lebron, S., Dumitrescu, A. S., Watkins, T. B. K., Uebele, V. N., et al. (2013). Calcineurin signaling mediates activity-dependent relocation of the axon initial segment. J. Neurosci. 33, 6950-6963. doi: 10.1523/ JNEUROSCI.0277-13.2013

Evans, M. D., Tufo, C., Dumitrescu, A. S., and Grubb, M. S. (2017). Myosin II activity is required for structural plasticity at the axon initial segment. Eur. J. Neurosci. 46, 1751-1757. doi: 10.1111/ejn.13597

Ford, M. C., Alexandrova, O., Cossell, L., Stange-Marten, A., Sinclair, J., KoppScheinpflug, C., et al. (2015). Tuning of Ranvier node and internode properties

\section{FUNDING}

This work was supported by the Dutch Fund for Economic Structure Reinforcement (FES, 0908 'NeuroBasic PharmaPhenomics Project') and Netherlands Organisation for Scientific Research (NWO; 823.02.006).

\section{ACKNOWLEDGMENTS}

We want to thank Minetta Elgersma-Hooisma and Mehrnoush Aghadavoud Jolfaei for breeding and genotyping, Laura de Kok for help with image analysis, and Jay Couey for advice on adult slices.

in myelinated axons to adjust action potential timing. Nat. Commun. 6:8073. doi: $10.1038 /$ ncomms 9073

Forsythe, I. D. (1994). Direct patch recording from identified presynaptic terminals mediating glutamatergic EPSCs in the rat CNS, in vitro. J. Physiol. 479, 381-387. doi: 10.1113/jphysiol.1994.sp020303

Galiano, M. R., Jha, S., Ho, T. S., Zhang, C., Ogawa, Y., Chang, K. J., et al. (2012). A distal axonal cytoskeleton forms an intra-axonal boundary that controls axon initial segment assembly. Cell 149, 1125-1139. doi: 10.1016/j.cell.2012.03.039

Grande, G., and Wang, L.-Y. (2011). Morphological and functional continuum underlying heterogeneity in the spiking fidelity at the calyx of Held synapse in vitro. J. Neurosci. 31, 13386-13399. doi: 10.1523/JNEUROSCI.0400-11.2011

Grubb, M. S., and Burrone, J. (2010). Activity-dependent relocation of the axon initial segment fine-tunes neuronal excitability. Nature 465, 1070-1074. doi: $10.1038 /$ nature09160

Guinan, J. J. Jr., and Li, R. Y.-S. (1990). Signal processing in brainstem auditory neurons which receive giant endings (calyces of Held) in the medial nucleus of the trapezoid body of the cat. Hear. Res. 49, 321-334. doi: 10.1016/03785955(90)90111-2

Gulledge, A. T., and Bravo, J. J. (2016). Neuron morphology influences axon initial segment plasticity. eNeuro 3:ENEURO.0085-15.2016. doi: 10.1523/ENEURO. 0085- 15.2016

Gutzmann, A., Ergül, N., Grossmann, R., Schultz, C., Wahle, P., and Engelhardt, M. (2014). A period of structural plasticity at the axon initial segment in developing visual cortex. Front. Neuroanat. 8:11. doi: 10.3389/fnana.2014.00011

Hamada, M. S., Goethals, S., De Vries, S. I., Brette, R., and Kole, M. H. P. (2016). Covariation of axon initial segment location and dendritic tree normalizes the somatic action potential. Proc. Natl. Acad. Sci. U.S.A. 113, 14841-14846. doi: $10.1073 /$ pnas. 1607548113

Hope, K. A., Ledoux, M. S., and Reiter, L. T. (2017). Glial overexpression of Dube3a causes seizures and synaptic impairments in Drosophila concomitant with down regulation of the $\mathrm{Na}^{+} / \mathrm{K}^{+}$pump ATP $\alpha$. Neurobiol. Dis. 108, 238-248. doi: 10.1016/j.nbd.2017.09.003

Huang, H. S., Burns, A. J., Nonneman, R. J., Baker, L. K., Riddick, N. V., Nikolova, V. D., et al. (2013). Behavioral deficits in an Angelman syndrome model: effects of genetic background and age. Behav. Brain Res. 243, 79-90. doi: 10.1016/j.bbr. 2012.12.052

Jiang, Y. H., Armstrong, D., Albrecht, U., Atkins, C. M., Noebels, J. L., Eichele, G., et al. (1998). Mutation of the Angelman ubiquitin ligase in mice causes increased cytoplasmic p53 and deficits of contextual learning and long-term potentiation. Neuron 21, 799-811. doi: 10.1016/S0896-6273(00) 80596-6

Johnston, J., Forsythe, I. D., and Kopp-Scheinpflug, C. (2010). Going native: voltage-gated potassium channels controlling neuronal excitability. J. Physiol. 588, 3187-3200. doi: 10.1113/jphysiol.2010.191973

Joshi, I., and Wang, L.-Y. (2002). Developmental profiles of glutamate receptors and synaptic transmission at a single synapse in the mouse auditory brainstem. J. Physiol. 540, 861-873. doi: 10.1113/jphysiol.2001.013506

Judson, M. C., Wallace, M. L., Sidorov, M. S., Burette, A. C., Gu, B., Van Woerden, G. M., et al. (2016). GABAergic neuron-specific loss of Ube3a causes Angelman 
syndrome-like EEG abnormalities and enhances seizure susceptibility. Neuron 90, 56-69. doi: 10.1016/j.neuron.2016.02.040

Kaphzan, H., Buffington, S. A., Jung, J. I., Rasband, M. N., and Klann, E. (2011). Alterations in intrinsic membrane properties and the axon initial segment in a mouse model of Angelman syndrome. J. Neurosci. 31, 17637-17648. doi: 10.1523/jneurosci.4162-11.2011

Kaphzan, H., Buffington, S. A., Ramaraj, A. B., Lingrel, J. B., Rasband, M. N., Santini, E., et al. (2013). Genetic reduction of the $\alpha 1$ subunit of $\mathrm{Na} / \mathrm{K}$-ATPase corrects multiple hippocampal phenotypes in Angelman syndrome. Cell Rep. 4, 405-412. doi: 10.1016/j.celrep.2013.07.005

Kim, S. E., Turkington, K., Kushmerick, C., and Kim, J. H. (2013). Central dysmyelination reduces the temporal fidelity of synaptic transmission and the reliability of postsynaptic firing during high-frequency stimulation. J. Neurophysiol. 110, 1621-1630. doi: 10.1152/jn.00117.2013

Koike-Tani, M., Kanda, T., Saitoh, N., Yamashita, T., and Takahashi, T. (2008). Involvement of AMPA receptor desensitization in short-term synaptic depression at the calyx of Held in developing rats. J. Physiol. 586, 2263-2275. doi: 10.1113/jphysiol.2007.142547

Kuba, H., Adachi, R., and Ohmori, H. (2014). Activity-dependent and activityindependent development of the axon initial segment. J. Neurosci. 34, 34433453. doi: 10.1523/jneurosci.4357-13.2014

Kuba, H., Ishii, T. M., and Ohmori, H. (2006). Axonal site of spike initiation enhances auditory coincidence detection. Nature 444, 1069-1072. doi: 10.1038/ nature 05347

Kuba, H., and Ohmori, H. (2009). Roles of axonal sodium channels in precise auditory time coding at nucleus magnocellularis of the chick. J. Physiol. 587, 87-100. doi: 10.1113/jphysiol.2008.162651

Kuba, H., Oichi, Y., and Ohmori, H. (2010). Presynaptic activity regulates $\mathrm{Na}^{+}$ channel distribution at the axon initial segment. Nature 465, 1075-1078. doi: $10.1038 /$ nature 09087

Kuba, H., Yamada, R., Ishiguro, G., and Adachi, R. (2015). Redistribution of Kv1 and Kv7 enhances neuronal excitability during structural axon initial segment plasticity. Nat. Commun. 6:8815. doi: 10.1038/ncomms9815

Leão, R. M., Kushmerick, C., Pinaud, R., Renden, R., Li, G.-L., Taschenberger, H., et al. (2005). Presynaptic $\mathrm{Na}^{+}$channels: locus, development, and recovery from inactivation at a high-fidelity synapse. J. Neurosci. 25, 3724-3738. doi: 10.1523/ JNEUROSCI.3983-04.2005

Leão, R. N., Leão, R. M., Da Costa, L. F., Rock Levinson, S., and Walmsley, B. (2008). A novel role for MNTB neuron dendrites in regulating action potential amplitude and cell excitability during repetitive firing. Eur. J. Neurosci. 27, 3095-3108. doi: 10.1111/j.1460-9568.2008.06297.x

Leão, R. N., Naves, M. M., Leão, K. E., and Walmsley, B. (2006). Altered sodium currents in auditory neurons of congenitally deaf mice. Eur. J. Neurosci. 24, 1137-1146. doi: 10.1111/j.1460-9568.2006.04982.x

Li, W., Yao, A., Zhi, H., Kaur, K., Zhu, Y. C., Jia, M., et al. (2016). Angelman syndrome protein Ube3a regulates synaptic growth and endocytosis by inhibiting BMP signaling in Drosophila. PLOS Genet. 12:e1006062. doi: 10.1371/ journal.pgen.1006062

Lorteije, J. A. M., and Borst, J. G. G. (2011). Contribution of the mouse calyx of Held synapse to tone adaptation. Eur. J. Neurosci. 33, 251-258. doi: 10.1111/j. 1460-9568.2010.07507.x

Lorteije, J. A. M., Rusu, S. I., Kushmerick, C., and Borst, J. G. G. (2009). Reliability and precision of the mouse calyx of Held synapse. J. Neurosci. 29, 13770-13784. doi: 10.1523/JNEUROSCI.3285-09.2009

Mathews, P. J. (2008). Voltage Gated ion Channels Shape Subthreshold Synaptic Integration in Principal Neurons of the Medial Superior Olive. Ph.D. thesis, The University of Texas at Austin, Austin, TX.

Mertz, L. G. B., Christensen, R., Vogel, I., Hertz, J. M., Nielsen, K. B., Grønskov, K., et al. (2013). Angelman syndrome in Denmark. Birth incidence, genetic findings, and age at diagnosis. Am. J. Med. Genet. A 161A, 2197-2203. doi: 10.1002/ajmg.a.36058

Ming, G., and Wang, L. Y. (2003). Properties of voltage-gated sodium channels in developing auditory neurons of the mouse in vitro. Chin. Med. Sci. J. 18, 67-74.

Morest, D. K. (1968). The growth of synaptic endings in the mammalian brain: a study of the calyces of the trapezoid body. Z. Anat. Entwicklungsgesch. 127, 201-220. doi: 10.1007/BF00526129

Nagtegaal, A. P., Spijker, S., Crins, T. T. H., Consortium, N.-B. M. P., and Borst, J. G. G. (2012). A novel QTL underlying early-onset, low-frequency hearing loss in BXD recombinant inbred strains. Genes Brain Behav. 11, 911-920. doi: 10.1111/j.1601-183X.2012.00845.x

Nozari, M., Suzuki, T., Rosa, M. G. P., Yamakawa, K., and Atapour, N. (2017). The impact of early environmental interventions on structural plasticity of the axon initial segment in neocortex. Dev. Psychobiol. 59, 39-47. doi: 10.1002/dev. 21453

Poliak, S., Gollan, L., Martinez, R., Custer, A., Einheber, S., Salzer, J. L., et al. (1999). Caspr2, a new member of the neurexin superfamily, is localized at the juxtaparanodes of myelinated axons and associates with $\mathrm{K}^{+}$channels. Neuron 24, 1037-1047. doi: 10.1016/S0896-6273(00)81049-1

Renden, R., Taschenberger, H., Puente, N., Rusakov, D. A., Duvoisin, R., Wang, L.-Y., et al. (2005). Glutamate transporter studies reveal the pruning of metabotropic glutamate receptors and absence of AMPA receptor desensitization at mature calyx of Held synapses. J. Neurosci. 25, 8482-8497. doi: 10.1523/JNEUROSCI.1848-05.2005

Rodríguez-Contreras, A., Van Hoeve, J. S., Habets, R. L. P., Locher, H., and Borst, J. G. G. (2008). Dynamic development of the calyx of Held synapse. Proc. Natl. Acad. Sci. U.S.A. 105, 5603-5608. doi: 10.1073/pnas.0801395105

Scott, L. L., Hage, T. A., and Golding, N. L. (2007). Weak action potential backpropagation is associated with high-frequency axonal firing capability in principal neurons of the gerbil medial superior olive. J. Physiol. 583, 647-661. doi: 10.1113/jphysiol.2007.136366

Scott, R. S., Henneberger, C., Padmashri, R., Anders, S., Jensen, T. P., and Rusakov, D. A. (2014). Neuronal adaptation involves rapid expansion of the action potential initiation site. Nat. Commun. 5:3817. doi: 10.1038/ncomms4817

Sierksma, M. C., and Borst, J. G. G. (2017). Resistance to action potential depression of a rat axon terminal in vivo. Proc. Natl. Acad. Sci. U.S.A. 114, 4249-4254. doi: $10.1073 /$ pnas. 1619433114

Silva-Santos, S., Van Woerden, G. M., Bruinsma, C. F., Mientjes, E., Jolfaei, M. A., Distel, B., et al. (2015). Ube3a reinstatement identifies distinct developmental windows in a murine Angelman syndrome model. J. Clin. Invest. 125, 2069-2076. doi: 10.1172/JCI80554

Tan, M. L., and Borst, J. G. G. (2007). Comparison of responses of neurons in the mouse inferior colliculus to current injections, tones of different durations, and sinusoidal amplitude-modulated tones. J. Neurophysiol. 98, 454-466. doi: $10.1152 /$ jn. 00174.2007

Taschenberger, H., Leão, R. M., Rowland, K. C., Spirou, G. A., and Von Gersdorff, H. (2002). Optimizing synaptic architecture and efficiency for highfrequency transmission. Neuron 36, 1127-1143. doi: 10.1016/S0896-6273(02) 01137-6

Taschenberger, H., Scheuss, V., and Neher, E. (2005). Release kinetics, quantal parameters and their modulation during short-term depression at a developing synapse in the rat CNS. J. Physiol. 568, 513-537. doi: 10.1113/jphysiol.2005. 093468

Ting, J. T., Daigle, T. L., Chen, Q., and Feng, G. (2014). Acute brain slice methods for adult and aging animals: application of targeted patch clamp analysis and optogenetics. Methods Mol. Biol. 1183, 221-242. doi: 10.1007/978-1-49391096-0_14

Trunova, S., Baek, B., and Giniger, E. (2011). Cdk5 regulates the size of an axon initial segment-like compartment in mushroom body neurons of the Drosophila central brain. J. Neurosci. 31, 10451-10462. doi: 10.1523/jneurosci.0117-11. 2011

Valdez, C., Scroggs, R., Chassen, R., and Reiter, L. T. (2015). Variation in Dube3a expression affects neurotransmission at the Drosophila neuromuscular junction. Biol. Open 4, 776-782. doi: 10.1242/bio.20148045

Van Woerden, G. M., Harris, K. D., Hojjati, M. R., Gustin, R. M., Qiu, S., De Avila Freire, R., et al. (2007). Rescue of neurological deficits in a mouse model for Angelman syndrome by reduction of $\alpha$ CaMKII inhibitory phosphorylation. Nat. Neurosci. 10, 280-282. doi: 10.1038/nn1845

Varela, J. A., Sen, K., Gibson, J., Fost, J., Abbott, L. F., and Nelson, S. B. (1997). A quantitative description of short-term plasticity at excitatory synapses in layer 2/3 of rat primary visual cortex. J. Neurosci. 17, 7926-7940.

Wallace, M. L., Burette, A. C., Weinberg, R. J., and Philpot, B. D. (2012). Maternal loss of Ube3a produces an excitatory/inhibitory imbalance through neuron type-specific synaptic defects. Neuron 74, 793-800. doi: 10.1016/j.neuron.2012. 03.036

Wallace, M. L., Van Woerden, G. M., Elgersma, Y., Smith, S. L., and Philpot, B. D. (2017). Ube3a loss increases excitability and blunts orientation tuning in the 
visual cortex of Angelman syndrome model mice. J. Neurophysiol. 118, 634-646. doi: 10.1152/jn.00618.2016

Wang, T., De Kok, L., Willemsen, R., Elgersma, Y., and Borst, J. G. G. (2015). In vivo synaptic transmission and morphology in mouse models of Tuberous sclerosis, Fragile X syndrome, Neurofibromatosis type 1, and Costello syndrome. Front. Cell. Neurosci. 9:234. doi: 10.3389/fncel.2015.00234

Wang, T., Rusu, S. I., Hruskova, B., Turecek, R., and Borst, J. G. G. (2013). Modulation of synaptic depression of the calyx of Held synapse by GABAB receptors and spontaneous activity. J. Physiol. 591, 4877-4894. doi: 10.1113/ jphysiol.2013.256875

Williams, C. A., Beaudet, A. L., Clayton-Smith, J., Knoll, J. H., Kyllerman, M., Laan, L. A., et al. (2006). Angelman syndrome 2005: updated consensus for diagnostic criteria. Am. J. Med. Genet. A 140, 413-418. doi: 10.1002/ajmg.a. 31074

$\mathrm{Xu}$, J., Berret, E., and Kim, J. H. (2016). Activity-dependent formation and location of voltage-gated sodium channel clusters at a CNS nerve terminal during postnatal development. J. Neurophysiol. 117, 582-593. doi: 10.1152/jn.00617.2016

Yamashita, T., Ishikawa, T., and Takahashi, T. (2003). Developmental increase in vesicular glutamate content does not cause saturation of AMPA receptors at the calyx of Held synapse. J. Neurosci. 23, 3633-3638.
Yang, Y. M., Wang, W., Fedchyshyn, M. J., Zhou, Z., Ding, J., and Wang, L. Y. (2014). Enhancing the fidelity of neurotransmission by activity-dependent facilitation of presynaptic potassium currents. Nat. Commun. 5:4564. doi: 10.1038/ncomms5564

Yu, Y., Shu, Y., and Mccormick, D. A. (2008). Cortical action potential backpropagation explains spike threshold variability and rapid-onset kinetics. J. Neurosci. 28, 7260-7272. doi: 10.1523/JNEUROSCI.1613-08.2008

Zheng, Q. Y., Johnson, K. R., and Erway, L. C. (1999). Assessment of hearing in 80 inbred strains of mice by ABR threshold analyses. Hear. Res. 130, 94-107. doi: 10.1016/S0378-5955(99)00003-9

Conflict of Interest Statement: The authors declare that the research was conducted in the absence of any commercial or financial relationships that could be construed as a potential conflict of interest.

Copyright (c) 2018 Wang, van Woerden, Elgersma and Borst. This is an open-access article distributed under the terms of the Creative Commons Attribution License (CC BY). The use, distribution or reproduction in other forums is permitted, provided the original author(s) or licensor are credited and that the original publication in this journal is cited, in accordance with accepted academic practice. No use, distribution or reproduction is permitted which does not comply with these terms. 